\title{
TOPOLOGY ON COHOMOLOGY OF LOCAL FIELDS
}

\author{
KĘSTUTIS ČESNAVIČIUS \\ Department of Mathematics, University of California, Berkeley, CA 94720-3840, USA; \\ email: kestutis@berkeley.edu
}

Received 17 December 2014; accepted 1 August 2015

\begin{abstract}
Arithmetic duality theorems over a local field $k$ are delicate to prove if char $k>0$. In this case, the proofs often exploit topologies carried by the cohomology groups $H^{n}(k, G)$ for commutative finite type $k$-group schemes $G$. These ‘Čech topologies', defined using Čech cohomology, are impractical due to the lack of proofs of their basic properties, such as continuity of connecting maps in long exact sequences. We propose another way to topologize $H^{n}(k, G)$ : in the key case when $n=1$, identify $H^{1}(k, G)$ with the set of isomorphism classes of objects of the groupoid of $k$-points of the classifying stack $\mathbf{B} G$ and invoke Moret-Bailly's general method of topologizing $k$-points of locally of finite type $k$-algebraic stacks. Geometric arguments prove that these 'classifying stack topologies' enjoy the properties expected from the Čech topologies. With this as the key input, we prove that the Čech and the classifying stack topologies actually agree. The expected properties of the Čech topologies follow, and these properties streamline a number of arithmetic duality proofs given elsewhere.
\end{abstract}

2010 Mathematics Subject Classification: 11S99 (primary); 11S25, 14A20 (secondary)

\section{Introduction}

1.1. The need for topology on cohomology. Let $k$ be a nonarchimedean local field of characteristic $p \geqslant 0$. The study of cohomology groups $H^{n}(k, G)$ for commutative finite $k$-group schemes $G$ is facilitated by Tate local duality (extended to the $p \mid \# G$ case in [Sha64]):

$$
H^{n}(k, G) \quad \text { and } \quad H^{2-n}\left(k, G^{D}\right) \quad \text { are Pontryagin duals, }
$$

(C) The Author 2015. This is an Open Access article, distributed under the terms of the Creative Commons Attribution licence (http://creativecommons.org/licenses/by/4.0/), which permits unrestricted re-use, distribution, and reproduction in any medium, provided the original work is properly cited. 
where $G^{D}$ is the Cartier dual of $G$. If $p \nmid \# G$, then $H^{n}(k, G)$ is finite, but if $p \mid \# G$ (when $H^{n}$ abbreviates $H_{\mathrm{fpp}}^{n}$ ), then such finiteness fails: for instance,

$$
H^{1}\left(\mathbb{F}_{p}((t)), \alpha_{p}\right) \cong \mathbb{F}_{p}((t)) / \mathbb{F}_{p}((t))^{p} .
$$

Therefore, in general one endows $H^{n}(k, G)$ with a topology and interprets ( $\left.\ddagger\right)$ as Pontryagin duality of Hausdorff locally compact abelian topological groups.

The above illustrates a general phenomenon: local arithmetic duality theorems face complications in positive characteristic due to the failure of finiteness of various cohomology groups. These complications are overcome by exploiting topologies carried by the relevant cohomology groups. The aim of this paper is to present a new way to define these topologies. This way is outlined in Section 1.3 and

(1) a priori seems more robust than the 'Čech cohomology approach' used elsewhere;

(2) a posteriori gives the same topology as the usual 'Čech cohomology approach'.

The interplay of (1) and (2) has particularly pleasant implications for local arithmetic duality theorems: as we explain in Section 1.2, it streamlines a number of proofs given elsewhere.

1.2. The Čech cohomology approach. This way to topologize $H^{n}(k, G)$, where $k$ is a nonarchimedean local field of characteristic $p>0$ and $G$ is a commutative finite type $k$-group scheme, is explained in [Mil06, III.Section 6]. It first establishes a connection with Čech cohomology:

$$
H^{n}(k, G)=\lim _{L} H^{n}(L / k, G),
$$

where $L / k$ runs over finite extensions in an algebraic closure $\bar{k}$, and then endows $H^{n}(L / k, G)$ with the subquotient topology of $G\left(\bigotimes_{i=0}^{n} L\right)$, and $H^{n}(k, G)$ with the direct limit topology. This 'Čech topology' on $H^{n}(k, G)$ is discussed further in Section 5.1.

A quotient topology or a direct limit topology can be difficult to work with individually, and the Čech topology, which combines them, seems particularly unwieldy. A number of its expected properties lack proofs; for example, it is not clear whether $H^{n}(k, G)$ is a topological group, nor whether $H^{n}(k, G)$ is discrete if $G$ is smooth and $n \geqslant 1$, nor whether connecting maps in long exact cohomology sequences are continuous. Such continuity is used in the proofs of local arithmetic duality theorems in positive characteristic; see, for instance, [Mil06, III.6.11 or 
III.7.8]. Not knowing it in general, one resorts to ad hoc modifications of these proofs to ensure their completeness.

In contrast, the way to topologize $H^{n}(k, G)$ outlined in Section 1.3 permits geometric proofs of all the desired properties, which illustrates (1). The corresponding properties of the Čech topology follow thanks to (2). This renders the ad hoc modifications mentioned earlier unnecessary.

1.3. The classifying stack approach. This way to topologize $H^{n}(k, G)$, where $k$ and $G$ are as in Section 1.2, uses the identification of $H^{1}(k, G)$ with the set of isomorphism classes of $G$-torsors, that is, the set of isomorphism classes of objects of the groupoid of $k$-points of the classifying stack $\mathbf{B} G$. In [MB01, Section 2], Moret-Bailly showed how to exploit the topologies on $X(k)$ for locally of finite type $k$-schemes $X$ to topologize the set of isomorphism classes of objects of $\mathscr{X}(k)$ for any locally of finite type $k$-algebraic stack $\mathscr{X}$ (see Section 2, especially Section 2.4, for details). His definition applies to $\mathscr{X}=\mathbf{B} G$ and hence topologizes $H^{1}(k, G)$. As for the other $H^{n}$, the identification $H^{0}(k, G)=G(k)$ should be the way to topologize $H^{0}$, whereas the topology on $H^{n}(k, G)$ for $n \geqslant 2$ could simply be defined to be discrete. The resulting 'classifying stack topology' on $H^{n}(k, G)$ seems convenient to work with for the following reasons.

(a) Its properties follow from geometric arguments; for example, connecting maps from $H^{0}$ to $H^{1}$ are continuous because they have underlying morphisms of algebraic stacks.

(b) Its definition permits other local topological rings (for example, the ring of integers of $k$ ) as bases, which allows us to treat all local fields simultaneously.

(c) Its definition permits $G$ that are locally of finite type, or noncommutative, or algebraic spaces, or all of these at once.

We exploit the properties of the classifying stack topology to prove in Remark 5.2 and Theorems 5.11 and 6.5 that this topology actually agrees with the Čech topology. In particular, the latter is discrete for $n \geqslant 2$; this seems not to have been noticed in the existing literature.

1.4. A summary of our conclusions. Due to (a)-(c), the classifying stack topology lends itself to general settings. For convenience of a reader not interested in generalities (which occupy Sections 3-4), we summarize our findings in the case of commutative finite type group schemes $G$ over local fields $k$.

The $H^{n}(k, G)$ are locally compact Hausdorff abelian topological groups that are discrete for $n \geqslant 2$. If $G$ is smooth (in particular, if char $k=0$ ), then $H^{n}(k, G)$ 
is discrete for $n \geqslant 1$. If $k$ is nonarchimedean, $\mathfrak{o}$ is its ring of integers, and $\mathcal{G}$ is a finite type flat $\mathfrak{o}$-model of $G$, then $H^{1}(\mathfrak{o}, \mathcal{G})$ is compact and

$$
H^{1}(\mathfrak{o}, \mathcal{G}) \rightarrow H^{1}(k, G)
$$

is continuous and open; if $\mathcal{G}$ is separated, then $H^{1}(\mathfrak{o}, \mathcal{G})$ is Hausdorff.

For a short exact sequence

$$
0 \rightarrow H \rightarrow G \rightarrow Q \rightarrow 0
$$

of commutative finite type $k$-group schemes, all the maps in its long exact cohomology sequence are continuous.

- If $H$ is smooth (respectively, proper), then

$$
G(k) \rightarrow Q(k)
$$

is open (respectively, closed).

- If $G$ is smooth (respectively, proper), then

$$
Q(k) \rightarrow H^{1}(k, H)
$$

is open (respectively, closed).

- If $Q$ is smooth (respectively, proper), then

$$
H^{1}(k, H) \rightarrow H^{1}(k, G)
$$

is open (respectively, closed).

- Finally,

$$
H^{1}(k, G) \rightarrow H^{1}(k, Q)
$$

is always open (but possibly not closed).

All the subsequent maps in the long exact cohomology sequence are both open and closed.

Thanks to Theorems 5.11 and 6.5, all the claims above, except possibly the ones involving $\mathfrak{o}$, also hold for the Čech topology; a number of them seem not to have appeared in the literature.

1.5. Étale-openness, proper-closedness, and finite-closedness. To take advantage of the flexibility provided by Section 1.3(a)-(b), for example, to be able to treat all local fields without distinguishing archimedean cases, we adopt the axiomatic approach when it comes to the relevant features of the base topological ring. The key definitions are the following. For a local topological ring $R$ such that $R^{\times} \subset R$ is open and is a topological group when endowed with the subspace topology, 
- $R$ is étale-open if every étale morphism of finite type $R$-schemes is open on $R$-points;

- $R$ is proper-closed if every proper morphism of finite type $R$-schemes is closed on $R$-points;

- $R$ is finite-closed if every finite morphism of finite type $R$-schemes is closed on $R$-points.

Every local field is étale-open and proper-closed (and hence also finite-closed). For further examples, as well as a more thorough discussion of these notions, see Sections 2.8 and 2.12 .

The axiomatic approach has the added benefit of producing interesting topologies on cohomology beyond the cases when the base is a local field of positive characteristic, or its algebraic extension, or its ring of integers. Other interesting examples in positive characteristic include fields endowed with a Henselian valuation of arbitrary rank, as well as their rings of integers. However, a reader who is only interested in local fields could simplify the discussion by restricting to local field bases throughout.

\section{Remarks.}

1.6. Philippe Gille and Laurent Moret-Bailly investigated a similar approach to topologizing cohomology groups and obtained the comparison Theorem 5.11 for ground fields $K$ as in Section 2.12(2). In Section 3.2, we adopt their definition of the topology on $H^{n}$ for $n \geqslant 2$ (initially we used the discrete topology for such $n$ ). This topology is discrete in many cases; see Proposition 3.5.

1.7. When all the groups involved are affine, properties of the Čech topology have also been investigated in [BT14]. (The article [BT14] uses a definition of the Čech topology that a priori differs from the one used here and in [Mil06]. Example 5.12 shows that the analog of [BT14, Theorem on page 562] becomes false for the Čech topology used here.) Although we have not been able to understand the arguments of [BT14] completely, the analogs of [BT14, 3.1, 4.2.1, 5.1, 5.1.2, and 5.1.3] for the classifying stack topology are special cases of the results of Sections 3-4; see Remark 4.6 for details. These analogs combine with Theorems 5.11 and 6.5 to give further analogs for the Čech topology defined in Section 5.1.

1.8. The contents of the paper. In Section 2, which may be consulted as needed, we investigate Moret-Bailly's method of topologizing groupoids of rational points of algebraic stacks and also discuss the axiomatic notions mentioned in 
Section 1.5. In Section 3 we study properties of the topologies defined using the classifying stack approach, and in Section 4 we study topological properties of maps arising from a short exact sequence. Although Sections 3-4 are short, they rely on Section 2 and Appendices A and B, which provide the underlying geometric arguments. Appendix A, included mostly for convenience, gathers known facts that concern classifying stacks and their interplay with short exact sequences. Appendix B removes diagonal quasi-compactness assumptions from several results in the algebraic space and stack literature. These improvements eliminate a number of quasi-compactness assumptions in Sections 3-4; other applications are discussed in Appendix B. The agreement of the classifying stack and the Čech topologies is the subject of Sections 5-6: Section 5 concerns $H^{1}$, and Section 6 deals with $H^{n}$ for $n \geqslant 2$.

1.9. Notation and conventions. For a field $F$, its algebraic closure $\bar{F}$ is chosen implicitly. For a scheme $S$, its fppf site $S_{\text {fppf }}$ is the category of $S$-schemes with the families of flat, locally of finite presentation, and jointly surjective morphisms as coverings. The small étale site is denoted by $S_{\text {ét }}$. The fppf topology is our default choice when taking quotients or considering torsors (synonymously, right torsors). Similarly for cohomology: $H^{n}$ abbreviates $H_{\mathrm{fppf}}^{n}$. Topology on $H^{n}$ in the absence of the 'Čech' subscript is always that of Sections 3.1-3.2. For us, compactness does not entail Hausdorffness, and 'locally compact' means that every point has a compact neighborhood.

We follow the terminology of [SP] when dealing with algebraic spaces and stacks, albeit for brevity we call a morphism representable if it is representable by algebraic spaces. To emphasize the parallel with schemes, we denote fiber categories by $\mathscr{X}(S)$ instead of $\mathscr{X}_{S}$, and write $x \in \mathscr{X}(S)$ instead of $x \in \mathrm{Ob} \mathscr{X}(S)$. We write $\Delta_{\mathscr{X} / S}$ for the diagonal of an $S$-algebraic stack $\mathscr{X}$. As in [SP], $\Delta_{\mathscr{X} / S}$ is neither separated nor quasi-compact at the outset, so algebraic spaces need not be quasi-separated. This is useful: for example, if we were to insist on quasi-compact diagonals, then our results on the topology on $H^{1}(k, G)$ could not accommodate locally of finite type but not quasi-compact $G$. We ignore set-theoretic difficulties (inherently present in any discussion concerning algebraic stacks) that can be resolved by the use of universes or by the approach used in [SP].

\section{Topologizing $R$-points of algebraic stacks}

As we recall in Section 2.2, the topology on a Hausdorff topological field $K$ gives rise to a natural topology on $X(K)$ for every locally of finite type $K$ scheme $X$. In [MB01, Section 2], Moret-Bailly exhibited an elegant way to extend the definition of this topology to the case when $X$ is an algebraic stack. This 
extension is of major importance for us through the case of a classifying stack B $G$. Therefore, in Section 2.4 we review the definition of [MB01, Section 2] (in a slightly more general setting when the base topological ring $R$ need not be a field) and proceed to detail the properties of the resulting topologies in the remainder of Section 2. These properties, especially Proposition 2.9(a) and Proposition 2.14, are key for our work in Sections 3-4.

2.1. The topology on $R$. Throughout Section $2, R$ denotes a local topological ring that satisfies the following.

( $\alpha$ ) The group of units $R^{\times}$is open in $R$ (equivalently, the maximal ideal $\mathfrak{m} \subset R$ is closed).

( $\beta$ ) The inverse map $R^{\times} \stackrel{x \mapsto x^{-1}}{\longrightarrow} R^{\times}$is continuous when $R^{\times} \subset R$ is endowed with the subspace topology.

Examples of $R$ that will be of main interest to us are Hausdorff topological fields and arbitrary valuation rings (endowed with their valuation topology).

2.2. The scheme case. For locally of finite type $R$-schemes $X$, we want to topologize $X(R)$ so that the following hold.

(i) An $R$-morphism $X \rightarrow X^{\prime}$ induces a continuous $X(R) \rightarrow X^{\prime}(R)$.

(ii) For each $n \geqslant 0$, the identification $\mathbb{A}^{n}(R)=R^{n}$ is a homeomorphism.

(iii) A closed immersion $X \hookrightarrow X^{\prime}$ induces an embedding $X(R) \hookrightarrow X^{\prime}(R)$.

(iv) An open immersion $X \hookrightarrow X^{\prime}$ induces an open embedding $X(R) \hookrightarrow X^{\prime}(R)$.

Claim 2.2.1. Such a topologization must also satisfy the following.

(v) The identifications

$$
\left(X^{\prime} \times_{X} X^{\prime \prime}\right)(R)=X^{\prime}(R) \times_{X(R)} X^{\prime \prime}(R)
$$

are homeomorphisms.

Proof. The case when $X=\operatorname{Spec} R$ with $X^{\prime}$ and $X^{\prime \prime}$ affine is settled by (ii)-(iii), which show that any choice of closed immersions

$$
i^{\prime}: X^{\prime} \hookrightarrow \mathbb{A}^{n^{\prime}} \text { and } i^{\prime \prime}: X^{\prime \prime} \hookrightarrow \mathbb{A}^{n^{\prime \prime}}
$$

induces topological embeddings

$$
i^{\prime}(R): X^{\prime}(R) \hookrightarrow R^{n^{\prime}}, \quad i^{\prime \prime}(R): X^{\prime \prime}(R) \hookrightarrow R^{n^{\prime \prime}},
$$


and

$$
\left(i^{\prime} \times{ }_{R} i^{\prime \prime}\right)(R):\left(X^{\prime} \times_{R} X^{\prime \prime}\right)(R) \hookrightarrow R^{n^{\prime}+n^{\prime \prime}} .
$$

In the case when $X=\operatorname{Spec} R$, we begin by covering $X^{\prime}$ and $X^{\prime \prime}$ by open affines: $X^{\prime}=\bigcup_{i} U_{i}^{\prime}$ and $X^{\prime \prime}=\bigcup_{j} U_{j}^{\prime \prime}$. Then we recall that $R$ is local to obtain the bijections

$$
X^{\prime}(R)=\bigcup_{i} U_{i}^{\prime}(R), \quad X^{\prime \prime}(R)=\bigcup_{j} U_{j}^{\prime \prime}(R),
$$

and

$$
\left(X^{\prime} \times_{R} X^{\prime \prime}\right)(R)=\bigcup_{i, j}\left(U_{i}^{\prime} \times_{R} U_{j}^{\prime \prime}\right)(R),
$$

which are covers by open subsets due to (iv). We conclude by using the previous case.

In the general case, both the diagonal $\Delta_{X / R}$ and its base change

$$
X^{\prime} \times_{X} X^{\prime \prime} \hookrightarrow X^{\prime} \times_{R} X^{\prime \prime}
$$

are immersions. Thus, by (iii)-(iv), $\left(X^{\prime} \times_{X} X^{\prime \prime}\right)(R)$ has the subspace topology of $\left(X^{\prime} \times{ }_{R} X^{\prime \prime}\right)(R)$. However, by the $X=\operatorname{Spec} R$ case, this subspace topology is the topology of $X^{\prime}(R) \times_{X(R)} X^{\prime \prime}(R)$.

Uniqueness of the sought topologization is ensured by (ii)-(iv), whereas [Con12, Proposition 3.1] supplies the existence. Conditions $(\alpha)$ and $(\beta)$ are crucial for the existence: along with the requirement that $R$ is local, they ensure that the topologies on $R$-points interact well with glueing along opens.

Loc. cit. and [Con12, Proposition 2.1] also give further useful properties of the resulting topologies.

(vi) If $R$ is Hausdorff, then a closed immersion $X \hookrightarrow X^{\prime}$ induces a closed embedding $X(R) \hookrightarrow X^{\prime}(R)$.

(vii) If $R$ is locally compact and Hausdorff, then $X(R)$ is locally compact.

Let $R^{\prime}$ be another local topological ring satisfying $(\alpha)-(\beta)$, and let $R \stackrel{h}{\rightarrow} R^{\prime}$ be a continuous homomorphism.

(viii) Each map $X(R) \rightarrow X\left(R^{\prime}\right)$ is continuous.

(ix) If $h$ is an open embedding, then each $X(R) \rightarrow X\left(R^{\prime}\right)$ induced by $h$ is open.

(x) If $h$ is local and a closed embedding, then each $X(R) \rightarrow X\left(R^{\prime}\right)$ induced by $h$ is closed. 
Proof of (viii)-(x). By considering affine open covers as in the proof of (v), all the claims reduce to the case of an affine $X$. The affine case is in turn addressed in [Con12, Ex. 2.2].

REMARK 2.2.2. Although loc. cit. shows that for affine $X$ the condition that $h$ was local in (x) is not needed, the condition cannot be dropped in general. Namely, let $k$ be a nonarchimedean local field, $\mathfrak{o}$ its ring of integers, and $h$ the closed embedding $\mathfrak{o} \hookrightarrow k$. Build $X$ by glueing the Néron lft model of $\left(\mathbb{G}_{m}\right)_{k}$ over $\mathfrak{o}$ with $\mathbb{A}_{k}^{1}$ along $\left(\mathbb{G}_{m}\right)_{k} \subset \mathbb{A}_{k}^{1}$. Then $X(\mathfrak{o}) \rightarrow X(k)$ is the inclusion $k^{\times} \hookrightarrow k$, which is not closed.

2.3. The algebraic space case. Locally of finite type $R$-algebraic spaces $X$ are in particular algebraic stacks, so we use the procedure described in Section 2.4 to topologize $X(R)$.

2.4. The stack case. For a locally of finite type $R$-algebraic stack $\mathscr{X}$, we declare a subcategory $U \subset \mathscr{X}(R)$ to be open if it is full, stable under isomorphisms in $\mathscr{X}(R)$, and, for every $R$-morphism $f: X \rightarrow \mathscr{X}$ with $X$ a locally of finite type $R$ scheme, $f(R)^{-1}(U)$ is open in $X(R)$ (the latter is topologized as in Section 2.2). In the last condition, due to (iv), one can also restrict to affine $X$.

The above definition topologizes $\mathscr{X}(R)$ in the sense that arbitrary unions and finite intersections of opens are open. In particular, the set of isomorphism classes of objects of $\mathscr{X}(R)$ becomes a bona fide topological space, which, thanks to (i), is none other than that of Section 2.2 if $\mathscr{X}$ is representable by a scheme. The topology on this set of isomorphism classes uniquely recovers the collection of open subcategories, and hence also the 'topology', of $\mathscr{X}(R)$. When discussing the latter we freely use evident analogs of familiar notions (for example, closedness or continuity), which always correspond to bona fide notions after taking isomorphism classes of objects.

Some of the properties (i)-(x) have analogs for algebraic stacks; see Corollary 2.7 and Proposition 2.9(b), (c), and (e).

2.5. The presheaf case. For a set-valued presheaf $\mathscr{F}$ on the category of locally of finite type $R$-schemes, we declare $U \subset \mathscr{F}(R)$ to be open if, for every presheaf morphism $f: X \rightarrow \mathscr{F}$ with $X$ a locally of finite type $R$-scheme, $f(R)^{-1}(U)$ is open in $X(R)$. Similarly to Section 2.4 , this topologizes $\mathscr{F}(R)$. A presheaf morphism $\mathscr{F} \rightarrow \mathscr{F}^{\prime}$ induces a continuous $\mathscr{F}(R) \rightarrow \mathscr{F}^{\prime}(R)$. If $\mathscr{F}$ is the presheaf of isomorphism classes of objects of fiber categories of a locally of finite type $R$-algebraic stack $\mathscr{X}$, then the resulting topology on $\mathscr{F}(R)$ agrees with that of Section 2.4, and hence also with that of Section 2.2 if $\mathscr{X}$ is a scheme (that is, if $\mathscr{F}$ is representable). 
Our main case of interest is $\mathscr{F}=H^{n}(-, G)$ for an $n \in \mathbb{Z}_{\geqslant 0}$ and a commutative flat locally of finite presentation $R$-group algebraic space $G$; in this case, see Proposition 3.5(b)-(d) and Proposition 3.6 for conditions that ensure that $\mathscr{F}(R)$ is a topological group.

In the study of openness or closedness of induced maps on $R$-points, the following lemma often provides a robust reduction to the algebraic space case.

LEMMA 2.6. For a representable morphism $f: \mathscr{X} \rightarrow \mathscr{Y}$ of locally of finite type $R$-algebraic stacks and an $R$-morphism $s: Y \rightarrow \mathscr{Y}$ with $Y$ a finite type affine $R$-scheme, consider the Cartesian square

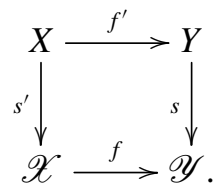

If $f^{\prime}(R)$ is open (respectively, closed) for every s as above, then $f(R)$ is open (respectively, closed).

Proof. For an open (respectively, closed) $U \subset \mathscr{X}(R)$, its full essential image $f(R)(U) \subset \mathscr{Y}(R)$ is open (respectively, closed) if so is $s(R)^{-1}(f(R)(U))$ for every $s$. It remains to observe the equality

$$
s(R)^{-1}(f(R)(U))=f^{\prime}(R)\left(s^{\prime}(R)^{-1}(U)\right)
$$

that is provided by the Cartesian property of (2.6.1).

COROLlary 2.7. The topologies of Section 2.4 satisfy the analogs of (i), (iv), (vi), and (viii).

Proof. Lemma 2.6 gives (iv) and (vi); (viii) follows from the scheme case; (i) is clear.

2.8. Étale-openness. Let $R$ be a local topological ring that satisfies $(\alpha)-(\beta)$. We call $R$ étale-open if, for every étale morphism $f: X \rightarrow Y$ of locally of finite type $R$-schemes, $f(R)$ is open. Due to (iv), the further requirement that $X$ and $Y$ are affine results in the same class of étale-open $R$.

Before proceeding, we give some examples of étale-open $R$; all $R$ in the examples are Henselian.

(1) $\mathbb{R}$ and $\mathbb{C}$.

(2) The fraction field $K$ of a Henselian valuation ring $A$. 
Proof. If $A=K$, then $K$ and $Y(K)$ are discrete. If $A \neq K$, then, according to [GGMB14, 3.1.4], for every étale morphism $f: X \rightarrow Y$ between finite type $K$-schemes, the induced morphism $f(K): X(K) \rightarrow Y(K)$ is a local homeomorphism; in particular, $f(K)$ is open.

(3) A Henselian valuation ring $A$.

Proof. We use (iv) to assume that $X$ and $Y$ are affine, and we let $K$ denote the fraction field of $A$. By (viii)-(ix), both $X(A) \hookrightarrow X(K)$ and $Y(A) \hookrightarrow Y(K)$ are inclusions of open subsets, so the openness of $f(A): X(A) \rightarrow Y(A)$ results from that of $f(K): X(K) \rightarrow Y(K)$ supplied by (2).

(4) A nonarchimedean local field and its ring of integers.

Proof. These are special cases of (2) and (3).

Due to the étale-local nature of algebraic spaces and the smooth-local nature of algebraic stacks, the étale-openness condition is key for the topologies on $R$ points of algebraic stacks to be well behaved. This condition is often accompanied by the requirement that $R$ is Henselian, which through Corollary B.7 ensures that $R$-points lift to some smooth scheme neighborhood (such liftings facilitate reductions to scheme cases). We record some of the resulting desirable properties in the following proposition.

Proposition 2.9. Let $h: R \rightarrow R^{\prime}$ be a continuous homomorphism between Henselian local topological rings that satisfy $(\alpha)-(\beta)$ and are étale-open, and let $\mathscr{X}, \mathscr{Y}$ be locally of finite type $R$-algebraic stacks such that $\Delta_{\mathscr{X} / R}$ has a separated $R$-fiber over the closed point of Spec $R$.

(a) If $f: \mathscr{X} \rightarrow \mathscr{Y}$ is a smooth morphism, then $f(R)$ is open.

(b) If $\Delta_{\mathscr{Y} / R}$ also has a separated $R$-fiber over the closed point of $\operatorname{Spec} R$, then the identification

$$
\left(\mathscr{X} \times_{R} \mathscr{Y}\right)(R) \stackrel{\sim}{\longrightarrow} \mathscr{X}(R) \times \mathscr{Y}(R)
$$

is a homeomorphism (compare with (v)).

(c) If $R$ is locally compact and Hausdorff, then $\mathscr{X}(R)$ is locally compact (compare with (vii)).

(d) If $R$ is compact Hausdorff and the number of isomorphism classes of objects of $\mathscr{X}(R / \mathfrak{m})$ is finite, where $\mathfrak{m} \subset R$ is the maximal ideal, then $\mathscr{X}(R)$ is compact. 
(e) If $h$ is an open embedding, then the map

$$
\mathscr{X}(R) \rightarrow \mathscr{X}\left(R^{\prime}\right)
$$

induced by $h$ is open (compare with (ix)).

Proof. We use Corollary B.7 to choose a smooth $\mathscr{X}$-scheme $X$ with

$$
X(R) \rightarrow \mathscr{X}(R)
$$

essentially surjective; for (d), we choose $X$ to be, in addition, affine.

(a) We use the continuity and the essential surjectivity of $X(R) \rightarrow \mathscr{X}(R)$ to replace $\mathscr{X}$ by $X$, and then we use Lemma 2.6 to assume that $\mathscr{Y}$ is a scheme and $\mathscr{X}$ is an algebraic space. We then once more replace $\mathscr{X}$ by $X$ to reduce to the case when both $\mathscr{X}$ and $\mathscr{Y}$ are schemes.

Due to (iv), we may work locally on $\mathscr{X}$. Therefore, due to [BLR90, Section 2.2/11], we may assume that $f$ factors as $\mathscr{X} \stackrel{g}{\rightarrow} \mathbb{A}_{\mathscr{Y}}^{n} \stackrel{\pi}{\rightarrow} \mathscr{Y}$, where $g$ is étale and $\pi$ is the canonical projection. It remains to observe that $g(R)$ is open by the étale-openness of $R$, and that $\pi(R)$ is a projection onto a direct factor by $(\mathrm{v})$, and hence is also open.

(b) We use Corollary B.7 to choose a smooth $\mathscr{Y}$-scheme $Y$ with $Y(R) \rightarrow \mathscr{Y}(R)$ essentially surjective. By the aspect (i) of Corollary 2.7, the identification in question is continuous. By (a), it inherits openness from the homeomorphism

$$
\left(X \times_{R} Y\right)(R) \stackrel{\sim}{\longrightarrow} X(R) \times Y(R) .
$$

(c) The claim follows by combining (a) applied to $X \rightarrow \mathscr{X}$ with (vii) applied to $X$.

(d) Since $X$ is affine, $X(R)$ is compact by (ii) and (vi); thus, so is its continuous image $\mathscr{X}(R)$.

(e) By (ix) applied to $X$, the map $X(R) \rightarrow X\left(R^{\prime}\right)$ induced by $h$ is open. By (a), the maps

$$
X(R) \rightarrow \mathscr{X}(R) \quad \text { and } \quad X\left(R^{\prime}\right) \rightarrow \mathscr{X}\left(R^{\prime}\right)
$$

are also open; moreover, $X(R) \rightarrow \mathscr{X}(R)$ is essentially surjective by construction. Therefore, by combining these observations, the map $\mathscr{X}(R) \rightarrow \mathscr{X}\left(R^{\prime}\right)$ induced by $h$ is open, too. 
2.10. Restriction of scalars. Let $R$ and $R^{\prime}$ be local topological rings that satisfy $(\alpha)-(\beta)$, and let $h: R \rightarrow R^{\prime}$ be a continuous homomorphism that makes $R^{\prime}$ a finite free $R$-module (both algebraically and topologically). Let $\mathscr{X}^{\prime}$ be a locally of finite type $R^{\prime}$-algebraic stack, and let

$$
\mathscr{X}:=\operatorname{Res}_{R^{\prime} / R} \mathscr{X}^{\prime}
$$

be its restriction of scalars defined as the category fibered in groupoids over Spec $R$ with $S$-points $\mathscr{X}^{\prime}\left(S_{R^{\prime}}\right)$, functorially in the $R$-scheme $S$. Assume that $\mathscr{X}$ is a locally of finite type $R$-algebraic stack. By [Ols06, 1.5], this assumption is met if $\mathscr{X}^{\prime}$ is locally of finite presentation over $R^{\prime}$ and $\Delta_{\mathscr{X}^{\prime} / R^{\prime}}$ is finite; by [SP, 05YF and 04AK] (compare with [BLR90, 7.6/4-5]), it is also met if $\mathscr{X}^{\prime}$ is an algebraic space that is locally of finite presentation over $R^{\prime}$.

We wish to describe some situations in which the identification

$$
\mathscr{X}^{\prime}\left(R^{\prime}\right) \cong \mathscr{X}(R)
$$

is a homeomorphism.

Proposition 2.11. In Section 2.10, the identification

$$
\iota: \mathscr{X}^{\prime}\left(R^{\prime}\right) \cong \mathscr{X}(R)
$$

is a homeomorphism

(a) if $\mathscr{X}^{\prime}$ is a scheme; or

(b) if $R$ and $R^{\prime}$ are Henselian étale-open and $\Delta_{\mathscr{X}^{\prime} / R^{\prime}}$ has a separated $R^{\prime}$-fiber over the closed point of Spec $R^{\prime}$.

Proof. Let $x^{\prime} \in \mathscr{X}^{\prime}\left(R^{\prime}\right)$ be arbitrary.

(a) If $\mathscr{X}^{\prime}$ is affine, then let $\mathscr{X}^{\prime} \hookrightarrow \mathbb{A}_{R^{\prime}}^{n}$ be a closed immersion. By [BLR90, 7.6/2(ii)], the restriction of scalars $\mathscr{X} \hookrightarrow \mathbb{A}_{R}^{d n}$ with $d$ determined by

$$
R^{\prime} \cong R^{\oplus d}
$$

is also a closed immersion. Therefore, since the isomorphism $R^{\prime} \cong R^{\oplus d}$ agrees with the topologies, (ii) and (iii) settle the case of affine $\mathscr{X}^{\prime}$.

If $\mathscr{X}^{\prime}$ is arbitrary, then let $U^{\prime} \subset \mathscr{X}^{\prime}$ be an affine open through which $x^{\prime}$ factors. By [BLR90, 7.6/2(i)], the restriction of scalars $U \subset \mathscr{X}$ is an open immersion. Therefore, by the aspect (iv) of Corollary 2.7,

$$
U(R) \subset \mathscr{X}(R) \quad \text { and } \quad U^{\prime}\left(R^{\prime}\right) \subset \mathscr{X}^{\prime}\left(R^{\prime}\right)
$$


are inclusions of open subsets, so $\iota$ is a local homeomorphism at $x^{\prime}$ by the settled affine case. Since $\iota$ is bijective and $x^{\prime}$ was arbitrary, $\iota$ must be a homeomorphism.

(b) By Theorem B.5, $x^{\prime}$ lifts to an $\tilde{x}^{\prime} \in X^{\prime}\left(R^{\prime}\right)$ for some affine scheme $X^{\prime}$ equipped with a smooth morphism $f^{\prime}: X^{\prime} \rightarrow \mathscr{X}^{\prime}$. By the infinitesimal lifting criterion [LMB00, 4.15],

$$
\operatorname{Res}_{R^{\prime} / R} f^{\prime}: X \rightarrow \mathscr{X}
$$

is smooth. (The proof of loc. cit. makes no use of the general conventions of [LMB00] that the base schemes are quasi-separated and that the diagonals of algebraic stacks are separated and quasi-compact.) Thus, (a) and Proposition 2.9(a) show that $\iota$ is a local homeomorphism at $x^{\prime}$. Since $\iota$ is also bijective, it must be a homeomorphism.

In addition to étale-openness, another pleasant situation is when finite or, more generally, proper morphisms induce closed maps on $R$-points. We axiomatize this in the following notions.

2.12. Proper-closedness and finite-closedness. Let $R$ be a local topological ring that satisfies $(\alpha)-(\beta)$. We call $R$ finite-closed (respectively, proper-closed) if, for every finite (respectively, proper) morphism $f: X \rightarrow Y$ of locally of finite type $R$-schemes, $f(R)$ is closed. Thanks to (iv), these concepts are unaltered if one further requires $Y$ to be affine. A proper-closed $R$ is also finite-closed. An $R$ that is finite-closed (or proper-closed) is also Hausdorff: take $f$ to be the inclusion of the origin of $\mathbb{A}_{R}^{1}$.

LEMMA 2.12.1. If $k$ is a local field, then the following hold.

(a) The field $k$ is proper-closed (and hence also finite-closed).

(b) The topological space $X(k)$ is compact for every proper $k$-algebraic space $X$.

(c) The map $f(k)$ is closed for every proper representable morphism

$$
f: \mathscr{X} \rightarrow \mathscr{Y}
$$

of locally of finite type $k$-algebraic stacks.

If, in addition, $k$ is nonarchimedean and $\mathfrak{o}$ is its ring of integers, then (a) and (b) also hold with $\mathfrak{o}$ in place of $k$ (for (c), see Proposition 2.16). 
Proof. The key input is Chow's lemma due to Knutson [Knu71, IV.3.1]; we use its following form.

LEMma 2.12.2 [SP, 088U]. For a proper map $f: X \rightarrow Y$ of separated $k$ algebraic spaces of finite type, there is a proper map $g: X^{\prime} \rightarrow X$ of $k$-algebraic spaces such that $g^{-1}(U) \cong U$ for a dense open subspace $U \subset X$ and $f \circ g$ factors through a closed immersion into $\mathbb{P}_{Y}^{n}$ for some $n \geqslant 0$.

Lemma 2.12.2 suggests the following strategy, which we borrow from [Con12, proof of Proposition 4.4]: reduce the claim in question to the cases when $X$ is replaced by a relative projective space or the reduced closed subspace $Z \subsetneq X$ complementary to $U$. Since the underlying topological space of $X$ is Noetherian, iteration will shrink $Z$ to the empty space, leaving only the first case to consider.

(a) Assume that $Y$ in Lemma 2.12.2 is an affine scheme. Since

$$
X(k)=g(k)\left(X^{\prime}(k)\right) \cup Z(k),
$$

the strategy above and (v)-(vi) reduce closedness of $f(k)$ to that of the second projection

$$
\mathbb{P}^{n}(k) \times Y(k) \rightarrow Y(k) \text { for } n \geqslant 0,
$$

which follows from the compactness of $\mathbb{P}^{n}(k)$.

(b) Combine the strategy above, (2.12.3), and the compactness of $X^{\prime}(k)$ inherited from $\mathbb{P}^{n}(k)$.

(c) Combine Lemma 2.6 and the proof of (a).

We now turn to the variants for $\mathfrak{o}$ in place of $k$. All of them reduce to their counterparts for $k$.

(a) Fix a proper morphism $f: X \rightarrow Y$ of finite type o-schemes with $Y$ affine, so $Y(\mathfrak{o}) \subset Y(k)$ is a closed embedding by $(\mathrm{x})$ and Remark 2.2.2. By the valuative criterion,

$$
f(k)^{-1}(Y(\mathfrak{o}))=X(\mathfrak{o})
$$

in $X(k)$, so $\left.f(k)\right|_{X(\mathfrak{o})}=f(\mathfrak{o})$ by (ix).

(b) By the valuative criterion of properness for algebraic spaces [SP, 0A40], Corollary 2.7(viii), and Proposition 2.9(e) (with Section 2.8(3)),

$$
X(\mathfrak{o}) \rightarrow X(k)
$$

is a homeomorphism. If $X$ is a scheme, (ix) applies directly. 
Before proceeding, we give some examples of proper-closed and finiteclosed $R$.

(1) A local field $k$ is proper-closed. So is its ring of integers $\mathfrak{o}$ if $k$ is nonarchimedean.

Proof. This is a special case of Lemma 2.12.1.

(2) Let $A$ be a Henselian valuation $\operatorname{ring}, K:=\operatorname{Frac}(A)$, and $\widehat{K}$ the completion of $K$. If $\widehat{K} / K$ is a separable field extension, then both $A$ and $K$ are finiteclosed.

Proof. If $A=K$, then $K$ and $Y(K)$ are discrete. If $A \neq K$, then $K$ is finite-closed by [GGMB14, 4.2.6], and $A$ is then also finite-closed by the method of proof of Lemma 2.12.1(a) for $\mathfrak{o}$.

REMARK 2.13. The étale-open and finite-closed conditions have already appeared in the literature: they are properties $(\mathrm{H})$ and $(\mathrm{F})$ in [MB12, Section 2.1] in the case of a Hausdorff topological field.

In Propositions 2.14-2.16 we record several situations in which morphisms of algebraic stacks (for example, of schemes) induce closed maps on $R$-points. These results will be useful in Section 4; see the proof of Proposition 4.4. Proposition 2.14 allows quite general $R$ at the expense of stringent conditions on the morphism, whereas Propositions 2.15 and 2.16 allow more general morphisms but restrict $R$.

PROPOSITION 2.14. Let $R$ be a local topological ring that satisfies $(\alpha)-(\beta)$, and let

$$
f: \mathscr{X} \rightarrow \mathscr{Y}
$$

be a morphism of locally of finite type $R$-algebraic stacks.

(a) If $R$ is finite-closed and $f$ is finite (and hence representable by schemes), then $f(R)$ is a closed map.

(b) If $R$ is proper-closed and $f$ is proper and representable by schemes, then $f(R)$ is a closed map.

Proof. The claims follow from Lemma 2.6 and from the definitions.

PROPOSITION 2.15. Let $R$ be a local topological ring that is Hausdorff, satisfies $(\alpha)-(\beta)$, and such that $R^{\times}$is closed in $R$ (for example, $R$ could be a valuation ring). Let

$$
f: \mathscr{X} \rightarrow \mathscr{Y}
$$


be a representable by schemes morphism of locally of finite type $R$-algebraic stacks.

(a) If $f$ is a quasi-compact immersion, then $f(R)$ is a closed embedding.

(b) If $R$ is finite-closed and $f$ is quasi-finite, then $f(R)$ is a closed map.

(c) If $R$ is proper-closed and $f$ is of finite type, then $f(R)$ is a closed map.

Proof. In (a), we need to show that the continuous monomorphism $f(R)$ is closed. In all cases Lemma 2.6 permits us to assume that $\mathscr{Y}$ is an affine scheme Spec $B$ (so $f$ is a scheme morphism).

(a) By Section 2.2(vi), we may assume that $f$ is a quasi-compact open immersion. By quasi-compactness, we may further assume that $f$ is the inclusion of a principal open affine Spec $B[1 / b]$. We view $b$ as a morphism $b: \mathscr{Y} \rightarrow \mathbb{A}_{R}^{1}$. Then the closedness of $R^{\times}$implies that of

$$
f(R)(\mathscr{X}(R))=b(R)^{-1}\left(R^{\times}\right),
$$

so the claim results from Section 2.2(iv).

Thanks to the quasi-compactness of $f$, in the proof of (b) and (c) we may replace $\mathscr{X}$ by an open affine, and hence further assume that $f$ is separated.

(b) Combine [EGA IV $\mathbf{I V}_{4}, 18.12 .13$ ] (that is, Zariski's main theorem) and (a).

(c) Combine [Con07b, Theorem 4.1] (that is, Nagata's embedding theorem) and (a).

PROPOSITION 2.16. Let $\mathfrak{o}$ be the ring of integers of a nonarchimedean local field $k$. For every representable, separated, finite type morphism

$$
f: \mathscr{X} \rightarrow \mathscr{Y}
$$

of locally of finite type o-algebraic stacks, the map $f(\mathfrak{o})$ is closed.

Proof. Lemma 2.6 reduces to the case when $\mathscr{Y}$ is an affine scheme. Nagata's embedding theorem for algebraic spaces [CLO12, Theorem 1.2.1] and Proposition 2.15(a) reduce further to proper $f$. By Section 2.2(viii) and the first part of the first sentence of Remark 2.2.2, $\mathscr{Y}(\mathfrak{o}) \subset \mathscr{Y}(k)$ is a closed embedding. By the valuative criterion of properness for algebraic spaces [SP, 0A40],

$$
f(k)^{-1}(\mathscr{Y}(\mathfrak{o}))=\mathscr{X}(\mathfrak{o})
$$


in $\mathscr{X}(k)$, so the embedding $\mathscr{X}(\mathfrak{o}) \subset \mathscr{X}(k)$ that results from Proposition 2.9(e) has a closed image, and hence is a closed embedding. It remains to note that $f(k)$ is closed by Lemma 2.12.1(c).

We close Section 2 by surveying conditions which ensure that $\mathscr{X}(R)$ is Hausdorff.

Proposition 2.17. Let $R$ be a local topological ring that is Hausdorff and satisfies $(\alpha)-(\beta)$, and let $\mathscr{X}$ be a locally of finite type $R$-algebraic stack such that $\Delta_{\mathscr{X} / R}$ has a separated $R$-fiber over the closed point of Spec $R$. If

(1) $\mathscr{X}$ is a separated $R$-scheme; or

(2) $R^{\times}$is closed in $R$ (for example, $R$ is valuation ring), and $\mathscr{X}$ is a quasiseparated $R$-scheme;

then $\mathscr{X}(R)$ is Hausdorff. If $R$ is Henselian and étale-open and

(3) $\mathscr{X}$ is a separated $R$-algebraic space; or

(4) $R^{\times}$is closed in $R$, and $\mathscr{X}$ is a quasi-separated $R$-algebraic space with $\Delta_{\mathscr{X} / R}$ an immersion; or

(5) $R$ is finite-closed and $\Delta_{\mathscr{X} / R}$ is finite; or

(6) $R$ is finite-closed, $R^{\times}$is closed in $R$, and $\Delta_{\mathscr{X} / R}$ is quasi-finite and separated; or

(7) $R$ is a local field and $\mathscr{X}$ is $R$-separated (that is, $\Delta_{\mathscr{X} / R}$ is proper); or

(8) $R$ is the ring of integers of a nonarchimedean local field, and $\Delta_{\mathscr{X} / R}$ is quasicompact and separated; or

(9) $R$ is proper-closed, and $\Delta_{\mathscr{X} / R}$ is proper and representable by schemes; or

(10) $R$ is proper-closed, $R^{\times}$is closed in $R$, and $\Delta_{\mathscr{X} / R}$ is quasi-compact and representable by schemes;

then $\mathscr{X}(R)$ is Hausdorff.

Proof. The identification $\left(\mathscr{X} \times_{R} \mathscr{X}\right)(R) \stackrel{\sim}{\longrightarrow} \mathscr{X}(R) \times \mathscr{X}(R)$ is a homeomorphism:

- in cases (1)-(2), this follows from Section 2.2(v);

- in cases (3)-(10), this follows from Proposition 2.9(b). 
It remains to note that the diagonal map $\Delta_{\mathscr{X} / R}(R)$ is closed:

- in case (1), this follows from Section 2.2(vi);

- in cases (2) and (4), this follows from Proposition 2.15(a);

- in case (3), this follows from the aspect (vi) of Corollary 2.7;

- in case (5), this follows from Proposition 2.14(a);

- in case (6), this follows from Proposition 2.15(b) (representability of $\Delta_{\mathscr{X} / R}$ by schemes is guaranteed by [LMB00, A.2]);

- in case (7), this follows from Lemma 2.12.1(c);

- in case (8), this follows from Proposition 2.16 (quasi-compactness ensures that $\Delta_{\mathscr{X} / R}$ is of finite type because it is always locally of finite type by [SP, 04XS] or by [LMB00, 4.2 and its proof]);

- in case (9), this follows from Proposition 2.14(b);

- in case (10), this follows from Proposition 2.15(c) (supplemented by [SP, 04XS] or by [LMB00, 4.2] again).

\section{Topologies on cohomology sets via classifying stacks}

The setup introduced in Section 3.1 is valid throughout Section 3. Our main goal in Sections 3-4 is to detail the properties of the topologies introduced in Sections 3.1-3.2.

3.1. Topology on $H^{0}(R, G)$ and $H^{1}(R, G)$. Let $R$ be a local topological ring that satisfies $(\alpha)$ and $(\beta)$ of Section 2.1; that is, $R^{\times} \subset R$ is open and a topological group when endowed with the subspace topology. Let $G$ be a flat and locally of finite presentation $R$-group algebraic space, and let $\mathbf{B} G$ be its classifying stack, which is algebraic and $R$-smooth by Section A.1 and Proposition A.3. We use Section 2.4 to topologize

$$
H^{0}(R, G)=G(R) \quad \text { and } \quad H^{1}(R, G)
$$

by viewing the latter as the set of isomorphism classes of objects of $\mathbf{B} G(R)$. 
Although in most examples $G$ is a scheme, allowing algebraic spaces leads to a more robust theory and more flexible proofs: for instance, group algebraic spaces behave better than group schemes with respect to representability of quotients or of inner forms.

3.2. Topology on $H^{n}(R, G)$ for $n \geqslant 2$. If, in the setup of Section 3.1, $G$ is in addition commutative, then we use the definitions of Section 2.5 with $\mathscr{F}=H^{n}(-, G)$ to topologize

$$
H^{n}(R, G) \text { for } n \geqslant 2 .
$$

(One can also use Section 2.5 in the $n=0$ and $n=1$ cases: the discussion in Section 2.5 guarantees agreement with Section 3.1.)

The following two propositions illustrate the functoriality inherent in the definitions.

Proposition 3.3. For a homomorphism $G \rightarrow G^{\prime}$ between flat and locally of finite presentation $R$-group algebraic spaces, the induced maps

$$
H^{n}(R, G) \rightarrow H^{n}\left(R, G^{\prime}\right)
$$

are continuous for $n \leqslant 1$, and also for $n \geqslant 2$ if $G$ and $G^{\prime}$ are commutative.

Proof. There is an underlying presheaf morphism

$$
H^{n}(-, G) \rightarrow H^{n}\left(-, G^{\prime}\right),
$$

so the continuity follows from the discussion of Section 2.5.

Proposition 3.4. Let $T$ be a right $G$-torsor, and let ${ }_{T} G:=\operatorname{Aut}_{G}(T)$ be the corresponding inner form of $G$. Twisting by $T$ induces a homeomorphism

$$
H^{1}(R, G) \cong H^{1}\left(R,{ }_{T} G\right)
$$

that sends the class of $T$ to the neutral class.

Proof. By [Gir71, III.2.6.1(i)], twisting by $T$ induces an underlying isomorphism

$$
\mathbf{B} G \cong \mathbf{B}_{T} G
$$

of algebraic stacks over $R$. This isomorphism is defined by $X \mapsto \operatorname{Hom}_{G}(T, X)$, and hence sends the class of $T$ to the neutral class. 
Before proceeding to discuss finer topological properties, we record conditions which ensure discreteness of the topologies introduced in Sections 3.1-3.2.

Proposition 3.5. If $R$ is Henselian and étale-open (in the sense of Section 2.8), then

(a) $H^{1}(R, G)$ is discrete if $G$ is smooth;

(b) $H^{n}(R, G)$ is discrete for $n \geqslant 1$ if $G$ is commutative and smooth;

(c) $H^{n}(R, G)$ is discrete for $n \geqslant 2$ if $G$ is commutative and of finite presentation;

(d) $H^{n}(R, G)$ is discrete for $n \geqslant 2$ if $G$ is commutative and $R$ is excellent (and hence Noetherian).

Proof. We fix a locally of finite type $R$-scheme $X$, an $a \in H^{n}(R, G)$, and an $f \in H^{n}(X, G)$, which corresponds to a presheaf morphism $f: X \rightarrow H^{n}(-, G)$. We need to show that

$$
f(R)^{-1}(a) \subset X(R)
$$

is open. For every $x \in f(R)^{-1}(a)$, we will build an étale $g_{x}: U_{x} \rightarrow X$ such that

$$
x \in g_{x}(R)\left(U_{x}(R)\right) \text { and }\left.f\right|_{U_{x}}=\left.a\right|_{U_{x}} \text { in } H^{n}\left(U_{x}, G\right) .
$$

The latter forces

$$
g_{x}(R)\left(U_{x}(R)\right) \subset f(R)^{-1}(a),
$$

so the étale-openness of $R$ will prove the desired openness of $f(R)^{-1}(a) \subset X(R)$.

For the construction of $U_{x}$, we view $x$ as an $R$-morphism Spec $R \stackrel{x}{\rightarrow} X$, let $s \in \operatorname{Spec} R$ be the closed point, and let $\mathcal{O}$ be the Henselization of $X$ at $x(s)$. Since $R$ is Henselian, $x$ factors through $\operatorname{Spec} \mathcal{O} \rightarrow X$; being an $R$-morphism, $x$ also identifies the residue fields at $s$ and $x(s)$. Therefore,

$$
\left.f\right|_{\mathcal{O}}=\left.a\right|_{\mathcal{O}} \quad \text { in } H^{n}(\mathcal{O}, G)
$$

by Remark B.11 for (a), Theorem B.18 for (b), Proposition B.13 for (c), and [Toë11, 3.4] together with [EGA IV $\mathbf{I}_{2}, 7.8 .3$ ] and [EGA $\mathbf{I V}_{4}$, 18.7.6] for (d). It remains to employ limit formalism [SGA 4 ${ }_{\text {II }}$, VII, 5.9] to find a desired $g_{x}$ through which $\operatorname{Spec} \mathcal{O} \rightarrow X$ factors. (We use analogs of loc. cit. for algebraic spaces instead of schemes and fppf cohomology of algebraic spaces instead of étale cohomology of schemes. The proofs based on topos-theoretic generalities of [SGA $\left.4_{\text {II }}, \mathrm{VI}\right]$ are analogous, too.) 
In the remainder of Section 3 we suppress mentioning $H^{n}(R,-)$ for $n \geqslant 2$; in the cases of such $n$ we have nothing to add to the frequent discreteness given by Proposition 3.5(b)-(d).

For $n=0$ and $n=1$, the following conditions ensure that $H^{n}(R, G)$ is a topological group.

Proposition 3.6. Let $\mathfrak{m} \subset R$ be the maximal ideal.

(a) If $G$ is a scheme, then $H^{0}(R, G)$ is a topological group.

(b) If $R$ is Henselian and étale-open, then $H^{0}(R, G)$ is a topological group.

(c) If $R$ is Henselian and étale-open, $G$ is commutative, and either $G$ is smooth or $G_{R / \mathfrak{m}}$ is a scheme (by [Art69, 4.2], $G_{R / \mathfrak{m}}$ is a scheme if, for instance, it is quasi-separated), then $H^{1}(R, G)$ is an abelian topological group.

Proof. The inverse map of the group $H^{n}(R, G)$ is continuous because it is induced by an underlying morphism of algebraic stacks (even of schemes in (a) and of algebraic spaces in (b)), as mentioned in the last paragraph of Section A.1 in case (c). Likewise, we deduce that the multiplication map is also continuous once we argue that the bijection

$$
H^{n}(R, G \times G) \cong H^{n}(R, G) \times H^{n}(R, G)
$$

is a homeomorphism.

- In (a), (3.6.1) is a homeomorphism by Section 2.2(v).

- In (b), (3.6.1) is a homeomorphism by Proposition 2.9(b).

- In (c), if $G$ is smooth, then both sides of (3.6.1) are discrete by Proposition 3.5(a).

- In (c), if $G_{R / \mathfrak{m}}$ is a scheme, then $G_{R / \mathfrak{m}}$ is separated, so $\Delta_{\mathbf{B} G_{R / \mathfrak{m}}}$ is also separated by Lemma A.2(b), and hence (3.6.1) is a homeomorphism by Proposition 2.9(b).

In the following proposition we record conditions which ensure that $H^{n}(R, G)$ is locally compact. Local compactness (as well as Hausdorffness) is important to know in practice: for instance, Pontryagin duality concerns locally compact Hausdorff abelian topological groups, so to make sense of Tate-Shatz local duality over nonarchimedean local fields of positive characteristic one needs to know that the cohomology groups in question are locally compact and Hausdorff. 
Proposition 3.7. Suppose that $R$ is locally compact and Hausdorff.

(a) If $G$ is a scheme, then $H^{0}(R, G)$ is locally compact.

(b) If $R$ is Henselian and étale-open, then $H^{0}(R, G)$ is locally compact.

(c) If $R$ is Henselian and étale-open and $G_{R / \mathfrak{m}}$ is a scheme, then $H^{1}(R, G)$ is locally compact.

Proof.

(a) The claim is a special case of Section 2.2(vii).

(b) The claim is a special case of Proposition 2.9(c).

(c) If $G_{R / \mathfrak{m}}$ is a scheme, then it is separated, so $\Delta_{\mathbf{B} G_{R / \mathfrak{m}}}$ is also separated by Lemma A.2(b). The local compactness of $H^{1}(R, G)$ therefore follows from Proposition 2.9(c).

We proceed to record conditions that ensure Hausdorffness of $H^{1}(R, G)$. For conditions that ensure Hausdorffness of $H^{0}(R, G)$, see Proposition 2.17.

Proposition 3.8. Suppose that $R$ is Henselian and étale-open.

(1) If $G$ is smooth; or

(2) if $R$ is finite-closed (in the sense of Section 2.12) and $G$ is finite; or

(3) if $R$ is finite-closed, $R^{\times}$is closed in $R$, and $G$ is quasi-finite and separated; or

(4) if $R$ is a local field and $G$ is proper; or

(5) if $R$ is the ring of integers of a nonarchimedean local field and $G$ is quasicompact and separated; or

(6) if $R$ is proper-closed, $R^{\times}$is closed in $R$, and $G$ is quasi-affine;

then $H^{1}(R, G)$ is Hausdorff.

Proof. In case (1), the claim follows from Proposition 3.5(a). In cases (2)-(6), the claim follows from Proposition 2.17(5)-(8) and (10) (one also uses Lemma A.2(b)).

The following further Hausdorffness result was pointed out to us by Laurent Moret-Bailly. It proves in particular that $H^{1}(k, G)$ is Hausdorff if $k$ is a nonarchimedean local field and $G$ is a commutative $k$-group scheme of finite type. 
PROPOSITION 3.9. Let $k$ be the fraction field of a Henselian valuation ring, $\widehat{k}$ the completion of $k$, and $G$ a $k$-group scheme of finite type. Suppose that the field extension $\widehat{k} / k$ is separable. If the identity component $G^{\circ}$ is commutative or if $G_{\bar{k}}$ has no nonzero subtorus, then $H^{1}(k, G)$ is $\mathrm{T}_{1}$; that is, its points are closed. In particular, if $G$ is commutative, then $H^{1}(k, G)$ is Hausdorff.

Proof. If $G$ is commutative, then $H^{1}(k, G)$ is an abelian topological group by Proposition 3.6(c) (together with Section 2.8(2)), so the last sentence follows from the rest.

If $k$ is discrete, then so is $H^{1}(k, G)$. If $k$ is nondiscrete, then [GGMB14, Theorem 1.2(1)(c)] proves that for every finite type $k$-scheme $Y$ and every $G_{Y}$-torsor $f: X \rightarrow Y$ the image of the induced map

$$
f(k): X(k) \rightarrow Y(k)
$$

is closed. In other words, for every $k$-morphism $Y \stackrel{f}{\rightarrow} \mathbf{B} G$, the preimage of the neutral class of $\mathbf{B} G(k)$ is closed in $Y(k)$; that is, the neutral element of $H^{1}(k, G)$ is closed. The closedness of the other elements of $H^{1}(k, G)$ then follows from Proposition 3.4 because the assumptions on $G^{\circ}$ or on $G_{\bar{k}}$ may be checked after replacing $G$ by its base change to a finite subextension of $\bar{k} / k$ and are therefore inherited by the inner forms ${ }_{T} G$.

We close Section 3 with a result that describes topological properties of the pointed set $H^{1}(R, G)$ in the case when $R$ is the ring of integers of a nonarchimedean local field.

PROPOSITION 3.10. Let $k$ be a nonarchimedean local field, $\mathfrak{o}$ its ring of integers, $\mathbb{F}$ its residue field, and $G$ a flat and locally of finite type o-group algebraic space. The pullback map

$$
p: H^{1}(\mathfrak{o}, G) \rightarrow H^{1}(k, G)
$$

is continuous. If $G_{\mathbb{F}}$ is a scheme, then $p$ is open. If, in addition to $G_{\mathbb{F}}$ being a scheme, $G$ is quasi-compact (respectively, quasi-compact and separated), then $H^{1}(\mathfrak{o}, G)$ is compact (respectively, compact and Hausdorff).

Proof. The continuity of $p$ follows from the aspect (viii) of Corollary 2.7 applied to $X=\mathbf{B} G$. If $G_{\mathbb{F}}$ is a scheme, then $\Delta_{\mathbf{B} G_{\mathbb{F}}}$ is separated by Lemma A.2(b), and hence $p$ is open by Proposition 2.9(e). If $G_{\mathbb{F}}$ is a scheme and $G$ is quasicompact, then $G_{\mathbb{F}}$ is a group scheme of finite type over a finite field, so $H^{1}(\mathbb{F}, G)$ is finite and the compactness of $H^{1}(\mathfrak{o}, G)$ follows from Proposition 2.9(d). The Hausdorffness claim follows from Proposition 3.8(5). 


\section{Topological properties of maps in cohomology sequences}

4.1. The setup. As in Section 3, let $R$ be a local topological ring that satisfies $(\alpha)-(\beta)$ of Section 2.1. Similarly to Proposition A.4, let

$$
\iota: H \hookrightarrow G
$$

be a monomorphism of flat $R$-group algebraic spaces locally of finite presentation, use $\iota$ to identify $H$ with a subspace of $G$, and let

$$
Q:=G / H
$$

be the resulting quotient $R$-algebraic space, which is flat and locally of finite presentation by Proposition A.4(a) and [SP, 06ET and 06EV].

In this section, we investigate the topological properties of the maps in the long exact sequence

$$
\begin{aligned}
1 & \rightarrow H(R) \stackrel{\iota(R)}{\rightarrow} G(R) \rightarrow Q(R) \rightarrow H^{1}(R, H) \\
& \rightarrow H^{1}(R, G) \stackrel{x}{\rightarrow} H^{1}(R, Q) \stackrel{y}{\rightarrow} H^{2}(R, H) \stackrel{z}{\rightarrow} \cdots,
\end{aligned}
$$

where $x$ (respectively, $y$ ) is defined if $\iota(H)$ is normal (respectively, central) in $G$, and $z$ and the subsequent maps are defined if $G$ is commutative.

As in Section 3, we have nothing to add to Proposition 3.5(b)-(d) concerning the topological study of $H^{n}(R,-)$ with $n \geqslant 2$. Throughout Section 4 we therefore mostly omit such $n$ from consideration.

Proposition 4.2. All the maps in $(\star)$ are continuous (whenever defined).

Proof. All the maps have underlying morphisms of presheaves, so the discussion of Section 2.5 applies.

In Proposition 4.3 (respectively, Proposition 4.4), we record conditions which ensure that the maps appearing in the long exact sequence $(\star)$ are open (respectively, closed). Knowledge of these conditions will be handy in the proofs of the comparison with the Čech topology carried out in Sections 5-6.

Proposition 4.3. Suppose that $(R, \mathfrak{m})$ is Henselian and étale-open.

(a) If $H \rightarrow \operatorname{Spec} R$ is smooth, then

$$
G(R) \rightarrow Q(R)
$$

is open. 
(b) If $G \rightarrow \operatorname{Spec} R$ is smooth, then

$$
Q(R) \rightarrow H^{1}(R, H)
$$

is open.

(c) If $Q \rightarrow \operatorname{Spec} R$ is smooth and $H_{R / \mathrm{m}}$ is a scheme, then

$$
H^{1}(R, H) \rightarrow H^{1}(R, G)
$$

is open.

(d) If $\iota(H)$ is normal in $G$ and $G_{R / \mathfrak{m}}$ is a scheme, then

$$
H^{1}(R, G) \stackrel{x}{\rightarrow} H^{1}(R, Q)
$$

is open.

Proof. The claims follow by combining Proposition A.4 for $\mathcal{P}=$ 'smooth' and Proposition 2.9(a) (together with the last sentence of Remark B.6 to ensure that the diagonal assumption of Proposition 2.9 is met).

PROPOSITION 4.4. Consider the following conditions.

(1) $R$ is a local field.

(2) $R$ is the ring of integers of a nonarchimedean local field.

(3) $R$ is finite-closed.

(4) $R$ is proper-closed.

(5) $R$ is finite-closed and $R^{\times}$is closed in $R$.

(6) $R$ is proper-closed and $R^{\times}$is closed in $R$.

Then

(a) If $H \rightarrow \operatorname{Spec} R$ is proper (respectively, quasi-compact and separated, respectively, finite, respectively, proper and $G$ is a scheme, respectively, quasi-finite and $G$ is a scheme, respectively, quasi-compact and $G$ is a scheme) and $R$ satisfies (1) (respectively, (2), respectively, (3), respectively, (4), respectively, (5), respectively, (6)), then

$$
G(R) \rightarrow Q(R) \quad \text { is closed }
$$

(b) If $G \rightarrow \operatorname{Spec} R$ is proper (respectively, quasi-compact and separated, respectively, finite, respectively, proper, $Q$ is a scheme, and $H$ is a quasiaffine scheme, respectively, quasi-finite, $Q$ is a scheme, and $H$ is a quasiaffine scheme, respectively, quasi-compact, $Q$ is a scheme, and $H$ is a 
quasi-affine scheme) and $R$ satisfies (1) (respectively, (2), respectively, (3), respectively, (4), respectively, (5), respectively, (6)), then

$$
Q(R) \rightarrow H^{1}(R, H) \quad \text { is closed } .
$$

(c) If $Q \rightarrow \operatorname{Spec} R$ is proper (respectively, quasi-compact and separated, respectively, finite, respectively, quasi-finite and separated, respectively, quasi-affine) and $R$ satisfies (1) (respectively, (2), respectively, (3), respectively, (5), respectively, (6)), then

$$
H^{1}(R, H) \rightarrow H^{1}(R, G) \text { is closed }
$$

Proof. Due to Proposition A.4, depending on whether we are in the situation (a), or (b), or (c), the morphism $G \rightarrow Q$, or $Q \rightarrow \mathbf{B} H$, or $\mathbf{B} H \rightarrow \mathbf{B} G$ inherits the geometric properties imposed on $H$, or $G$, or $Q$, respectively. Therefore,

- in case (1), claims (a), (b), and (c) follow from Lemma 2.12.1(c);

- in case (2), claims (a), (b), and (c) follow from Proposition 2.16;

- in case (3), claims (a), (b), and (c) follow from Proposition 2.14(a);

- in case (4), claims (a) and (b) follow from Proposition 2.14(b) (for claim (b), one uses the quasi-affineness of $H$ through Lemma A.2(b) to argue that $\Delta_{\mathbf{B} H / R}$ is quasi-affine, so that $Q \rightarrow \mathbf{B} H$ is representable by schemes);

- in case (5), claims (a), (b), and (c) follow from Proposition 2.15(b), which applies because finite-closedness implies Hausdorffness (see Section 2.12), whereas the representability by schemes of the morphism in question is argued for claim (b) as in the proof of case (4), and is argued for claim (c) via the fact [LMB00, A.2] that a quasi-finite separated algebraic space over a scheme is a scheme;

- in case (6), claims (a), (b), and (c) follow from Proposition 2.15(c), which applies thanks to reasoning analogous to that of the proof of case (5).

\section{Remarks.}

4.5. Consider a single statement of Proposition 4.3 or Proposition 4.4, and let $t$ denote the map of $(\star)$ studied there. A continuous open (respectively, closed) surjection is a quotient map, so, if in addition to the assumptions of the statement in question also the map of $(\star)$ succeeding $t$ is defined and vanishes, then the target of $t$ inherits a quotient topology from the source. 
4.6. The approach of [BT14] is based on an analog of Remark 4.5 as follows. The article [BT14] assumes that $R$ is the fraction field of a complete rank-one valuation ring and $H$ is affine, embeds $H$ into a suitable smooth $G$ for which $H^{1}(R, G)$ vanishes (for example, into $\left.G L_{n}\right)$, and endows $H^{1}(R, H)$ with the resulting quotient topology. It then argues in [BT14, 2.1.1] that the choice of $G$ did not matter and claims in [BT14, 3.1] that the quotient topology agrees with the Čech topology (defined in [BT14, 2.2]). Conclusions about the Čech topology use this analysis; an analog of Proposition 4.2 is [BT14, 5.1], that of Proposition 4.3(c) is [BT14, 5.1.2], and that of Proposition 3.5(a) is [BT14, 5.1.3]. However, as mentioned in Remark 1.7, the Čech topology used in [BT14] is a priori different from the one used here and in [Mil06, III, Section 6], so we cannot use the results cited above to shorten the proofs given in Sections 5-6 below.

4.7. In contrast to Proposition 4.3(d), $x$ need not be closed even if $R$ is a local field and $H, G$, and $Q$ are commutative and finite. For example, consider

$$
0 \rightarrow \alpha_{p} \rightarrow \alpha_{p^{2}} \rightarrow \alpha_{p} \rightarrow 0
$$

over $R:=\mathbb{F}_{p}((t))$. The vanishing of $H^{n}\left(R, \mathbb{G}_{a}\right)$ for $n \geqslant 1$ combines with Remark 4.5 to show that

$$
H^{1}\left(R, \alpha_{p^{2}}\right)=R / R^{p^{2}} \text { and } H^{1}\left(R, \alpha_{p}\right)=R / R^{p}
$$

both algebraically and topologically, and, moreover, that $x$ identifies with the quotient map

$$
R / R^{p^{2}} \rightarrow R / R^{p}
$$

The latter is not closed:

$$
\left\{t^{n}+t^{-p n}\right\}_{n \geqslant 1 \text { and } p \nmid n} \subset R
$$

has a closed image in $R / R^{p^{2}}$ but not in $R / R^{p}$. Also, the $p$-power map $R / R^{p} \rightarrow R / R^{p^{2}}$ is not open, so the smoothness assumption of Proposition 4.3(c) cannot be dropped. Neither can that of Proposition 4.3(b) because $R / R^{p}$ is nondiscrete.

\section{Comparison with the Čech topology on $H^{1}(k, G)$}

The main goal of the present section is Theorem 5.11, which shows that the topology on $H^{1}(k, G)$ introduced in Section 3.1 agrees with the Čech topology used by other authors. 
5.1. The Čech topology. Let $k$ be a Hausdorff topological field and $G$ a locally of finite type $k$-group scheme. For a finite subextension $\bar{k} / L / k$ and $n \geqslant 0$, set

$$
\left.L_{n}:=\bigotimes_{i=0}^{n} L \quad \text { (tensor product over } k\right)
$$

Consider the restriction of scalars

$$
C^{n}:=\operatorname{Res}_{L_{n} / k}\left(G_{L_{n}}\right) .
$$

By [EGA IV, $9.1 .5]$ and [CGP10, A.3.5], the connected components of $G$ are quasi-projective. Therefore, every finite set of points of $G_{L_{n}}$ is contained in an open affine, and hence $C^{n}$ is a locally of finite type $k$-group scheme by [BLR90, 7.6/4]. We call $C^{n}$ the scheme of $n$-cochains (for $G$ with respect to $L / k$ ). The coboundaries

$$
d^{n}: C^{n} \rightarrow C^{n+1},
$$

which are defined using the usual formulas, are morphisms of $k$-schemes; they are even group homomorphisms if $G$ is commutative. Let $e_{n}$ : Spec $k \rightarrow C^{n}$ be the unit section, and let

$$
Z^{n}:=C^{n} \times_{d^{n}, C^{n+1}, e_{n+1}} \operatorname{Spec} k
$$

be the scheme of $n$-cocycles (for $G$ with respect to $L / k$ ).

The group $C^{0}(k)$ acts on the right on $Z^{1}(k)$ by

$$
\left(z^{1}, c^{0}\right) \mapsto p_{1}^{*}(k)\left(c^{0}\right)^{-1} z^{1} p_{0}^{*}(k)\left(c^{0}\right),
$$

where $p_{i}^{*}: C^{0} \rightarrow C^{1}$ is the map induced by the $i$ th projection $\operatorname{Spec} L_{1} \rightarrow \operatorname{Spec} L$ (so $\left.d^{0}=\left(p_{1}^{*}\right)^{-1} p_{0}^{*}\right)$. We endow the orbit space

$$
H^{1}(L / k, G):=Z^{1}(k) / C^{0}(k)
$$

with the quotient topology. Its points correspond to $G$-torsors trivialized by $L / k$, so

$$
H^{1}(L / k, G) \hookrightarrow H^{1}(k, G) .
$$

If $L^{\prime}$ is contained in $L$, then the inclusion $H^{1}\left(L^{\prime} / k, G\right) \hookrightarrow H^{1}(L / k, G)$ is continuous because it is induced by a $k$-morphism $Z_{L^{\prime} / k}^{1} \rightarrow Z_{L / k}^{1}$. The transition maps in

$$
H^{1}(k, G)=\lim _{L} H^{1}(L / k, G)
$$

are therefore continuous, and we write $H^{1}(k, G)_{\text {Cech }}$ for $H^{1}(k, G)$ endowed with the direct limit topology. A subset $U \subset H^{1}(k, G)_{\text {Cech }}$ is open if and only if so is its preimage in every $Z^{1}(k)$. 
If $G$ is commutative, then $\left(C^{n}(k), d^{n}(k)\right)_{n \geqslant 0}$ is a complex of abelian groups. We endow its cohomology groups $H^{n}(L / k, G)$ with the subquotient topology. If $G$ is locally of finite type, then

$$
H^{n}(k, G)=\lim _{L} H^{n}(L / k, G)
$$

by [Sha72, page 208, Theorem 42], and we write $H^{n}(k, G)_{\text {Čech }}$ for $H^{n}(k, G)$ endowed with the direct limit topology. (Loc. cit. assumes that $G$ is of finite type, but the method of proof there continues to work for locally of finite type $G$ because such a $G$ is an extension of a smooth $k$-group by a finite $k$-group due to Lemma 5.8.)

The agreement of our definition of the Čech topology with the one used in [Mil06, III, Section 6] follows from Proposition 2.11(a). The absence of the Čech subscript indicates the topologies of Sections 3.1-3.2.

REMARK 5.2. The bijection

$$
H^{0}(k, G)_{\check{C} \text { ech }} \rightarrow H^{0}(k, G)
$$

is a homeomorphism. In fact, both topologies agree with the topology on $G(k)$ : for $H^{0}(k, G)_{\check{C} \text { ech }}$, this follows from Section 2.2(x); for $H^{0}(k, G)$, this follows from the definition given in Section 3.1.

LEMMA 5.3. The bijection

$$
b_{G}: H^{1}(k, G)_{\text {Čech }} \rightarrow H^{1}(k, G)
$$

is continuous.

Proof. We need to argue that for every $L$ the composite

$$
Z^{1}(k) \rightarrow H^{1}(k, G)_{\check{C} \text { ech }} \rightarrow H^{1}(k, G)
$$

is continuous (as before, $Z^{1}$ depends on $L$ ). For this, it suffices to exhibit an underlying morphism

$$
Z^{1} \stackrel{a}{\rightarrow} \mathbf{B} G .
$$

On the category of $k$-schemes, $Z^{1}$ represents the functor of 1-cocycles for $G$ with respect to $L / k$. The universal 1 -cocycle corresponding to $\mathrm{id}_{Z^{1}}$ gives rise to a torsor under $G_{Z^{1}} \rightarrow Z^{1}$, and this torsor corresponds to $a$.

COROLlaRY 5.4. If $k$ is étale-open and $G \rightarrow$ Spec $k$ is smooth, then $b_{G}$ is a homeomorphism and $H^{1}(k, G)$ Čech is discrete. 
Proof. By Proposition 3.5(a), $H^{1}(k, G)$ is discrete, so continuity of $b_{G}$ gives the claim.

LEMMA 5.5. For a homomorphism $f: H \rightarrow G$ of locally of finite type k-group schemes, the resulting morphism

$$
H^{1}(k, H)_{\check{C} \text { ech }} \rightarrow H^{1}(k, G)_{\check{C} \text { ech }}
$$

is continuous.

Proof. For a finite subextension $\bar{k} / L / k$, write $f_{Z^{1}}: Z_{H}^{1} \rightarrow Z_{G}^{1}$ for the $k$-morphism of schemes of 1-cocycles induced by $f$. The claim results from the commutativity of

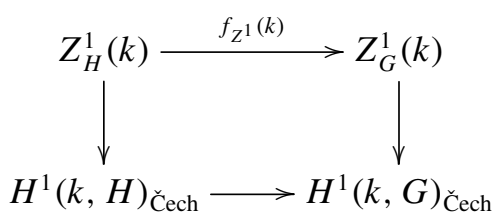

and the continuity of $f_{Z^{1}}(k)$.

Lemma 5.6. Let $T$ be a right $G$-torsor, and let ${ }_{T} G:=\operatorname{Aut}_{G}(T)$ be the corresponding inner form of $G$. Twisting by $T$ induces a homeomorphism

$$
H^{1}(k, G)_{\text {Čech }} \cong H^{1}\left(k,{ }_{T} G\right)_{\text {Čech }}
$$

that sends the class of $T$ to the neutral class.

Proof. We write $Z_{L / k, G}^{1}$ for the $Z^{1}$ of Section 5.1 and choose a finite subextension $\bar{k} / L / k$ that trivializes $T$ and an

$$
x \in Z_{L / k, G}^{1}(k)
$$

that gives the class of $T$ in $H^{1}(L / k, G)$. For every finite subextension $\bar{k} / L^{\prime} / L$, right multiplication by the pullback of $x^{-1}$ induces an isomorphism

$$
i: Z_{L^{\prime} / k, G}^{1} \stackrel{\sim}{\longrightarrow} Z_{L^{\prime} / k, T}^{1}
$$

(compare with [Ser02, I, Section 5, Proposition 35 bis]). Moreover, the homeomorphism $i(k)$ intertwines the actions of $C_{L^{\prime} / k, G}^{0}(k)$ and $C_{L^{\prime} / k, T}^{0}(k)$. Thus, the resulting 'twist by $T$ ' bijection

$$
H^{1}\left(L^{\prime} / k, G\right) \stackrel{\sim}{\longrightarrow} H^{1}\left(L^{\prime} / k,{ }_{T} G\right)
$$

is a homeomorphism. It remains to take $\lim _{L^{\prime}}$. 
LEMMA 5.7. Let $K$ be a field of characteristic $p$, and let $G$ be a finite connected $K$-group scheme.

(a) Every $G$-torsor $X \rightarrow \operatorname{Spec} K$ for the étale topology is trivial.

(b) For some $n \geqslant 0$ with $p^{n} \leqslant(\# G)$ !, every $G$-torsor $X \rightarrow$ Spec $K$ for the fppf topology is trivial over $K^{1 / p^{n}} \subset \bar{K}$ (with the convention that $0^{0}=1$ ). In particular, if $K$ is perfect, then $H^{1}(K, G)$ is trivial.

Proof. If $p=0$, then $G$ is trivial, so we assume that $p>0$.

(a) If $A$ is a reduced $K$-algebra, then $G(A)$ is a singleton. We take $A=L \bigotimes_{K} L$ for a finite separable extension $L / K$ to see that there are no nontrivial $G$ torsors trivialized by $L / K$.

(b) For a subextension $\bar{K} / K^{\prime} / K$ of degree at most \#G, let $N \subset \bar{K}$ be its normal closure, so $[N: K] \leqslant(\# G)$ !. Let $n$ be such that

$$
p^{n} \leqslant(\# G) ! \text { but } p^{n+1}>(\# G) ! .
$$

Set $L:=K^{1 / p^{n}}$.

If $K^{\prime}$ is a residue field of $X$, then, by [BouA, V.73, Proposition 13], $N L / L$ is separable. Thus, $X_{L}$ trivializes over an étale cover of Spec $L$. Since $G_{L}$ is connected, it remains to apply (a).

LEMMA 5.8. Let $K$ be a field. Every locally of finite type $K$-group scheme $G$ is an extension

$$
1 \rightarrow H \rightarrow G \rightarrow Q \rightarrow 1
$$

with $Q$ smooth and $H$ finite, connected, and closed and normal in $G$.

Proof. If char $K=0$, then $G$ is smooth by Cartier's theorem [SGA $3_{\text {I new }}, \mathrm{VI}_{\mathrm{B}}$, 1.6.1], so the trivial subgroup $H=1$ suffices. If char $K>0$, then [SGA $3_{\text {I new }}$, $\mathrm{VII}_{\mathrm{A}}, 8.3$ ] gives the claim (with $H$ a large enough relative Frobenius kernel).

5.9. The ground field. For the rest of Section $5, k$ is a Hausdorff topological field that is étale-open and finite-closed in the sense of Sections 2.8 and 2.12 and such that every finite extension $L$ of $k$ is finite-closed when endowed with the topology resulting from some (equivalently, any) $k$-module identification $L \cong k^{n}$. For instance, as justified in Sections 2.8 and 2.12, $k$ could be a local field or a field that is complete with respect to a nonarchimedean valuation of rank 1 . We set $p:=\operatorname{char} k$. 
Lemma 5.10. Assume that $\left[k: k^{p}\right]<\infty$ if $p>0$. For a locally of finite type $k$-group scheme $G$, a finite connected closed subgroup $H \leqslant G$ (that need not be normal), and $Q:=G / H$ (which is a locally of finite type $k$-scheme by [SGA $\left.3_{\text {I new }}, \mathrm{VI}_{\mathrm{A}}, 3.2\right]$ ), the connecting map

$$
\delta(k): Q(k) \rightarrow H^{1}(k, H)_{\text {Čech }}
$$

is continuous.

Proof. We use Lemma 5.7(b) to fix a finite subextension $\bar{k} / L / k$ for which $H^{1}(L / k, H) \hookrightarrow H^{1}(k, H)$ is bijective. We indicate the relevant groups by subscripts, and consider the Cartesian square

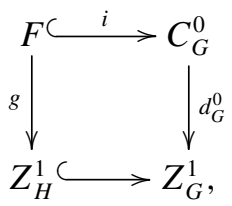

in which the horizontal maps are closed immersions thanks to [BLR90, 7.6/2(ii)]. Claim 5.10.1. There is a Cartesian square (for the bottom map, we recall the notation $C_{G}^{0}=\operatorname{Res}_{L / k}\left(G_{L}\right)$ )

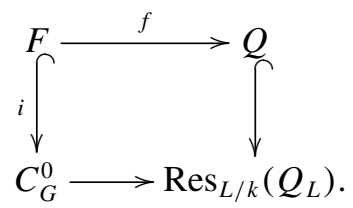

Proof. The claim is a manifestation of fppf descent: for a $k$-scheme $S$, the two pullbacks

$$
p_{0}^{*}(x), p_{1}^{*}(x) \in C_{G}^{1}(S)=G\left(S_{L} \times_{S} S_{L}\right) \quad \text { of an element } x \in C_{G}^{0}(S)=G\left(S_{L}\right)
$$

satisfy

$$
p_{0}^{*}(x)=p_{1}^{*}(x) y \quad \text { for some } y \in Z_{H}^{1}(S) \subset H\left(S_{L} \times_{S} S_{L}\right)
$$

if and only if the image of $x$ in $Q\left(S_{L}\right)$ lands in $Q(S)$.

Claim 5.10.3. $f(k): F(k) \rightarrow Q(k)$ is continuous, closed, and surjective.

Proof. Continuity is clear (from Section 2.2(i)). By the assumptions of Section $5.9, L$ is finite-closed, so

$$
G(L) \rightarrow Q(L)
$$


is closed. To deduce the same for $f(k)$, we evaluate (5.10.2) on $k$-points and apply Section 2.2(vi) to $i$, Proposition 2.11(a) to $C_{G}^{0}$, and Section 2.2(viii) to

$$
Q(k) \rightarrow Q(L) .
$$

By the choice of $L$, every $k$-point of $Q$ lifts to an $L$-point of $G$, so (5.10.2) also gives the surjectivity of $f(k)$.

Claim 5.10.4. The following diagram commutes:

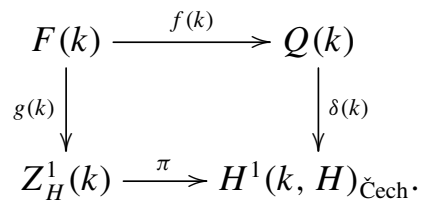

Proof. We fix a $q \in Q(k)$, let $X \rightarrow$ Spec $k$ be the corresponding fiber of $G \rightarrow Q$, and view $X$ as an $H$-torsor trivialized by $L / k$. To build a

$$
z \in Z_{H}^{1}(k) \subset H\left(L \bigotimes_{k} L\right)
$$

that gives the class of $X$ in $H^{1}(L / k, H)$, one takes any

$$
x \in X(L) \stackrel{(5.10 .2)}{\subset} F(k) \subset G(L)
$$

and sets $z:=d_{G}^{0}(x)$ to be the $H\left(L \bigotimes_{k} L\right)$-difference of the two pullbacks of $x$ to $X\left(L \bigotimes_{k} L\right)$. This gives the claim because $d_{G}^{0}(x)=g(k)(x)$.

To prove the continuity of $\delta(k)$, let $Z \subset H^{1}(k, H)_{\text {Čech }}$ be closed. The continuity of $\pi$ and $g(k)$ gives the closedness of

$$
g(k)^{-1}\left(\pi^{-1}(Z)\right) \stackrel{(5.10 .5)}{=} f(k)^{-1}\left(\delta(k)^{-1}(Z)\right),
$$

so Claim 5.10.3 gives that of $\delta(k)^{-1}(Z)$.

THEOREM 5.11. Let $k$ be a Hausdorff topological field of characteristic $p \geqslant 0$ such that $k$ is étale-open, every finite extension of $k$ endowed with the $k$-vector space topology is finite-closed, and $\left[k: k^{p}\right]<\infty$ if $p>0$. (For instance, $k$ could be a local field.) For a locally of finite type $k$-group scheme $G$, the bijection

$$
b_{G}: H^{1}(k, G)_{\check{C} \text { ech }} \rightarrow H^{1}(k, G)
$$

is a homeomorphism. 
Proof. We begin with the case of a finite connected $G$. We embed $G$ into some $\mathrm{GL}_{n}$, and set $Q:=\mathrm{GL}_{n} / G$. By Proposition 4.3(b) (with Remark 4.5), the connecting map

$$
\delta(k): Q(k) \rightarrow H^{1}(k, G)
$$

is a quotient map. On the other hand, by Lemma 5.10,

$$
\delta(k): Q(k) \rightarrow H^{1}(k, G)_{\text {Čech }}
$$

is continuous. Therefore,

$$
b_{G}: H^{1}(k, G)_{\check{C} e c h} \rightarrow H^{1}(k, G)
$$

is open, and hence is also a homeomorphism thanks to its continuity supplied by Lemma 5.3.

We now turn to the general case. By Lemma 5.8, there is an exact sequence

$$
1 \rightarrow H \rightarrow G \rightarrow Q \rightarrow 1
$$

with $H$ finite connected, $Q$ smooth, and $H$ closed and normal in $G$. Its cohomology sequence gives the commutative diagram



in which the horizontal maps are continuous by Proposition 4.2 and Lemma 5.5. By Corollary 5.4, $H^{1}(k, Q)_{\text {Čech }}$ is discrete, so the fibers of $\check{c}$ are open. By Lemma 5.3, $b_{G}$ is continuous, so it remains to argue that the restrictions of $b_{G}$ to the fibers of $\check{c}$ are open morphisms. In the case of the neutral fiber, the restriction $\left.b_{G}\right|_{\operatorname{Im} \check{a}}$ is open because $b_{H}$ is a homeomorphism by the finite connected case and $a$ is open by Proposition 4.3(c). We will argue that the openness of the restrictions of $b_{G}$ to the other fibers of $\check{c}$ follows from the settled case of the neutral fiber.

Let $T$ be a $G$-torsor, and let ${ }_{T} G:=\operatorname{Aut}_{G}(T)$ be the resulting inner form of $G$. By Proposition 3.4 and Lemma 5.6, twisting by $T$ induces homeomorphisms

$$
H^{1}(k, G)_{\text {Čech }} \cong H^{1}\left(k,{ }_{T} G\right)_{\text {Čech }}
$$

and

$$
H^{1}(k, G) \cong H^{1}\left(k,{ }_{T} G\right),
$$


which, moreover, are compatible with $b_{G}$ and $b_{T G}$. By [Gir71, III.3.3.5], these homeomorphisms identify the $\check{c}$-fiber containing the class of $T$ with the neutral fiber of

$$
H^{1}\left(k,{ }_{T} G\right)_{\text {Čech }} \rightarrow H^{1}\left(k,{ }_{T / H} Q\right)_{\text {Čech }},
$$

where $T / H$ is regarded as a $Q$-torsor and ${ }_{T / H} Q:=\operatorname{Aut}_{Q}(T / H)$ is the resulting inner form of $Q$. This identification achieves the desired reduction to the case of the neutral fiber because ${ }_{T / H} Q$ is smooth and $\operatorname{Ker}\left({ }_{T} G \rightarrow{ }_{T / H} Q\right)$ is finite connected, as may be checked after base change to a finite extension trivializing $T$.

Example 5.12. We show that the assumption on $\left[k: k^{p}\right]$ cannot be dropped in Theorem 5.11. We take

$$
k=\mathbb{F}_{p}\left(x_{1}, x_{2}, \ldots\right)((t)) \text { and } G=\alpha_{p} .
$$

Since $k$ is a complete discrete valuation ring, it satisfies the assumptions of Theorem 5.11 with the exception of the requirement on $\left[k: k^{p}\right]$. By Proposition 4.3(b) and Remark 4.5, $H^{1}(k, G)$ identifies with $k / k^{p}$ equipped with the quotient topology. In particular,

$$
\left\{x_{n} t^{n p}\right\}_{n \geqslant 1} \subset H^{1}(k, G)
$$

has 0 as a limit point, and hence is not closed. On the other hand, only finitely many $x_{n}$ have a $p$ th root in a fixed finite subextension $\bar{k} / L / k$, so the preimage of

$$
\left\{x_{n} t^{n p}\right\}_{n \geqslant 1} \subset H^{1}(k, G)_{\check{C} \text { ech }}
$$

in $H^{1}(L / k, G)$ is finite. Since $C^{0}(k)=\alpha_{p}(L)$ is finite, the further preimage in $Z^{1}(k)$ is also finite, and hence closed thanks to Proposition 2.17(1). In conclusion,

$$
\left\{x_{n} t^{n p}\right\}_{n \geqslant 1} \subset H^{1}(k, G)_{\text {Čech }}
$$

is closed, so $b_{G}$ is not a homeomorphism.

\section{Comparison with the Čech topology on $H^{n}(k, G)$ for $n \geqslant 2$}

The goal of Section 6 is Theorem 6.5: for commutative $G$ and $n \geqslant 2$, the Čech topology on $H^{n}(k, G)$ is simply the discrete topology, which by Proposition 3.5(b) agrees with the topology of Section 3.2.

6.1. The ground field. Throughout Section $6, k$ is a Hausdorff topological field that is étale-open in the sense of Section 2.8. For instance, $k$ could be a local field or the fraction field of a Henselian valuation ring. 
6.2. The Čech topology. Focussing on commutative $G$, we study the Čech topology defined in Section 5.1.

We begin with the analog of Corollary 5.4 in the commutative case.

LEMMA 6.3. If $G$ is a commutative smooth $k$-group scheme, then $H^{n}(k, G)_{\text {Čech }}$ is discrete for $n \geqslant 1$.

Proof. Fix a finite subextension $\bar{k} / L / k$. By Proposition 2.9(a) and Lemma B.15, the fibers of

$$
Z^{n}(k) \rightarrow H^{n}(L / k, G)
$$

are open. Thus, $H^{n}(L / k, G)$ is discrete, and hence so is $H^{n}(k, G)_{\text {Čech }}$.

LEMMA 6.4. For a field $K$, every commutative locally of finite type $K$-group scheme $H$ is a closed subgroup of a commutative smooth $K$-group scheme $G$, which can be chosen to be quasi-compact if so is $H$.

Proof. By Lemma 5.8, there is a short exact sequence

$$
0 \rightarrow H_{0} \rightarrow H \rightarrow H^{\prime} \rightarrow 0 \quad \text { with } H_{0} \text { finite connected and } H^{\prime} \text { smooth. }
$$

Let $H_{0}^{D}$ be the Cartier dual of $H_{0}$, so that $H_{0}=\mathcal{H o m}\left(H_{0}^{D}, \mathbb{G}_{m}\right)$. Embed $H_{0}$ into the smooth $k$-group $G_{0}:=\operatorname{Res}_{H_{0}^{D} / k} \mathbb{G}_{m}$ via the functor

$$
\mathcal{H o m}\left(H_{0}^{D}, \mathbb{G}_{m}\right) \hookrightarrow \mathcal{M o r}\left(H_{0}^{D}, \mathbb{G}_{m}\right)
$$

The pushout

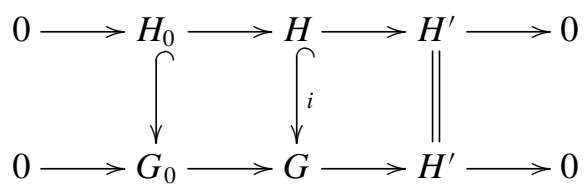

defines a desired $G$, because $G$ inherits $k$-smoothness from $G_{0}$ and $H^{\prime}$.

THEOREM 6.5. If $H$ is a commutative locally of finite type $k$-group scheme, then $H^{n}(k, H)_{\text {Čech }}$ is discrete for $n \geqslant 2$.

Proof. When needed, we indicate the relevant groups and subextensions by subscripts.

Fix an $a \in H^{n}(k, H)_{\text {Čech }}$ and its preimage $b \in Z_{L / k, H}^{n}(k)$ for a sufficiently large $L$. Let $V \subset Z_{L / k, H}^{n}(k)$ be the preimage of an open $U \subset H^{n}(k, H)_{\check{C} \text { ech }}$. The 
preimage of $a+U$ is the open $b+V$. Thus, the translation by $a$ map is open, and we are reduced to proving the openness of $\{0\} \subset H^{n}(k, H)_{\text {Čech }}$.

Use Lemma 6.4 to embed $H$ into a commutative smooth $k$-group scheme $G$, and set $Q:=G / H$, which is a commutative smooth $k$-group scheme by [SGA $\left.3_{\text {I new }}, \mathrm{VI}_{\mathrm{A}}, 3.2\right]$. Let

$$
\delta: H^{n-1}(k, Q)_{\text {Čech }} \rightarrow H^{n}(k, H)_{\text {Čech }}
$$

be the connecting homomorphism. By Lemma 6.3, for a fixed finite subextension $\bar{k} / L / k$,

$$
X:=\operatorname{Ker}\left(Z_{Q}^{n-1}(k) \rightarrow H^{n-1}(k, Q)_{\text {Čech }}\right) \quad \text { is a nonempty open in } Z_{Q}^{n-1}(k) .
$$

Let $Y \subset C_{G}^{n-1}(k)$ be the preimage of $X \subset Z_{Q}^{n-1}(k)$. By [Mil06, proof of III.6.1], the nonempty

$$
d_{G}^{n-1}(k)(Y) \subset Z_{H}^{n}(k) \quad \text { lies in the preimage of } \delta(0) \in H^{n}(k, H)_{\text {Čech }} .
$$

Claim 6.5.2. $d_{G}^{n-1}(k)(Y)$ is open in $Z_{H}^{n}(k)$.

Proof. The proof is similar to that of Lemma 5.10. Define the $k$-scheme $F$ to be the fiber product

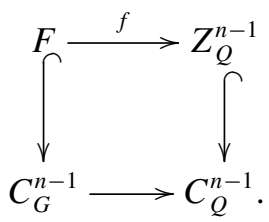

Then $Y=f(k)^{-1}(X)$, so the continuity of $f(k)$ guarantees the openness of $Y$ in $F(k)$.

Left exactness of the restriction of scalars and the definition of $F$ ensure that the outer square of

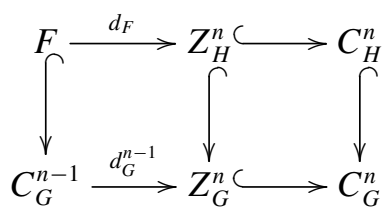

is Cartesian. Since so is the right square, the left one is also. Thus, $d_{F}$ is smooth because so is $d_{G}^{n-1}$ by Lemma B.15. Since

$$
d_{G}^{n-1}(k)(Y)=d_{F}(Y),
$$

it remains to combine the openness of $Y \subset F(k)$ with Proposition 2.9(a). 
Since $Z_{H}^{n}(k)$ is a topological group, (6.5.1) combines with Claim 6.5.2 to prove that the preimage of $\{0\} \subset H^{n}(k, H)_{\text {Cech }}$ in $Z_{H}^{n}(k)$ is open. But $L$ was arbitrary, so $\{0\} \subset H^{n}(k, H)_{\check{C} \text { ech }}$ is open.

\section{Acknowledgements}

I thank Laurent Moret-Bailly for suggesting a number of improvements to our initial results. I thank Bhargav Bhatt, Brian Conrad, Aise Johan de Jong, Bjorn Poonen, Yunqing Tang, and Bertrand Toën for helpful conversations or correspondence regarding the material of this paper. I thank the referees for helpful comments and suggestions.

\section{Appendix A. Generalities concerning stacks of torsors}

We gather generalities about the classifying stack $\mathbf{B} G$ that play an important role in the arguments of the main body of the paper. Specifically, we discuss algebraicity in Section A.1, properties of the diagonal in Lemma A.2 (which we use without explicit notice), smoothness in Proposition A.3, and properties of morphisms arising from a short exact sequence in Proposition A.4.

A.1. Stacks of torsors. Let $S$ be a scheme, and let $G$ be an $S$-group algebraic space. Consider the classifying $S$-stack $\mathbf{B} G$, whose groupoid of $S^{\prime}$-points for an $S$-scheme $S^{\prime}$ is the category of right torsors under $G_{S^{\prime}} \rightarrow S^{\prime}$ for the fppf topology (the torsors are algebraic spaces by [SP, 04SK], or already by [LMB00, 10.4.2] if $S$ and $G \rightarrow S$ are quasi-separated). By [SP, 06FI] (compare also [LMB00, 10.13.1]), $\mathbf{B} G$ is algebraic if $G \rightarrow S$ is flat and locally of finite presentation. Let $S \stackrel{e}{\rightarrow} \mathbf{B} G$ be the morphism that corresponds to the trivial torsor.

If $G$ is commutative, then the naturality of the contracted product and opposite torsor constructions shows that $\mathbf{B} G$ is a group object in the 2-category of stacks over $S$.

LEMmA A.2. Let $S$ be a scheme, and let $G$ be an $S$-group algebraic space.

(a) The following square is 2-Cartesian:




(b) Let $\mathcal{P}$ be a property of morphisms of algebraic spaces that is stable under base change and is fppf local on the base. If $G \rightarrow S$ has $\mathcal{P}$, then so do $\Delta_{\mathbf{B} G / S}$ and $S \stackrel{e}{\rightarrow} \mathbf{B} G$.

Proof.

(a) Inspect the definition of a 2-fiber product.

(b) Checking that $\Delta_{\mathbf{B} G / S}$ has $\mathcal{P}$ amounts to checking that, for every $S$-scheme $S^{\prime}$ and torsors $x$ and $y$ under $G_{S^{\prime}} \rightarrow S^{\prime}$, the algebraic space $\operatorname{Isom}_{G_{S^{\prime}}}(x, y) \rightarrow S^{\prime}$ parameterizing torsor isomorphisms has $\mathcal{P}$. $\operatorname{But}_{\operatorname{Isom}_{S^{\prime}}}(x, y) \rightarrow S^{\prime}$ is a right torsor for the fppf topology under an inner form of $G_{S^{\prime}} \rightarrow S^{\prime}$, and hence inherits $\mathcal{P}$. The claim that concerns $e$ now follows from (a).

Proposition A.3. Let $S$ be a scheme, and let $G$ be a flat and locally of finite presentation $S$-group algebraic space. The algebraic stack $\mathbf{B} G$ is $S$-smooth.

Proof. By Lemma A.2(b), $S \stackrel{e}{\rightarrow} \mathbf{B} G$ is faithfully flat and locally of finite presentation, so the algebraic stack analog of [SP, 05B5] (or of [EGA IV 17.7.7]) applies to

$$
S \stackrel{e}{\rightarrow} \mathbf{B} G \rightarrow S
$$

In the following result, $\mathcal{P}=$ 'smooth' is the case of most interest to us.

Proposition A.4. Let $S$ be a scheme, $H$ a flat and locally of finite presentation $S$-group algebraic space, $\iota: H \hookrightarrow G$ a monomorphism of $S$-group algebraic spaces, and $Q:=G / H$ the resulting quotient homogeneous space (which is an $S$ algebraic space by [SP, 06PH] or, in many situations, already by [LMB00, 8.1.1 and 10.6]). Let $\mathcal{P}$ be a property of morphisms of algebraic spaces that is stable under base change and is fppf local on the base.

(a) If $H \rightarrow S$ has $\mathcal{P}$, then

$$
G \rightarrow Q
$$

has $\mathcal{P}$.

(b) If $G \rightarrow S$ has $\mathcal{P}$, then the representable

$$
Q \rightarrow \mathbf{B} H
$$

that corresponds to $G \rightarrow Q$ has $\mathcal{P}$. 
(c) If $Q \rightarrow S$ has $\mathcal{P}$ and $G \rightarrow S$ is flat and locally of finite presentation, then

$$
\mathbf{B} H \stackrel{\mathbf{B} \iota}{\rightarrow} \mathbf{B} G
$$

is representable and has $\mathcal{P}$.

(d) If $\iota(H)$ is normal in $G$ and $G \rightarrow S$ is flat and locally of finite presentation, then

$$
\mathbf{B} G \rightarrow \mathbf{B} Q
$$

is smooth.

Proof. For (a), note that $G \rightarrow Q$ is a torsor under $H \times{ }_{S} Q \rightarrow Q$ for the fppf topology. In (b)-(d), the stacks in question are algebraic by the discussion in Section A.1 (for (d), $Q$ is flat and locally of finite presentation by (a) and [SP, 06ET and 06EV]). Moreover, by Lemma A.2(b), $e$ in
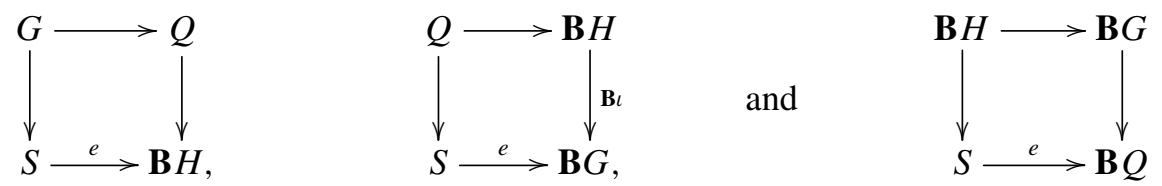

is flat and locally of finite presentation in cases (b), (c), and (d). Thus, to conclude using fppf descent (and Proposition A.3 for (d)), we argue that the squares are 2-Cartesian (in the respective cases).

(b) For a morphism $S^{\prime} \stackrel{x}{\rightarrow} Q$ with $S^{\prime}$ an $S$-scheme, a trivialization of the $H_{S^{\prime}}$ torsor $G \times_{Q, x} S^{\prime} \rightarrow S^{\prime}$ amounts to an element of $G\left(S^{\prime}\right)$ lifting $x$, functorially in $S^{\prime}$.

(c) For an $H$-torsor $T^{\prime}$, a trivialization the associated $G$-torsor $T$ amounts to an identification of $T^{\prime}$ with a sub- $H$-torsor of the trivial $G$-torsor, that is, an identification of $T^{\prime}$ with the fiber of $G \rightarrow Q$ above an $S$-point of $Q$, functorially in $S$.

(d) For a $G$-torsor $T$, a trivialization of the $Q$-torsor $T / H$ amounts to a sub- $H$ torsor $T^{\prime} \subset T$, that is, an $H$-torsor $T^{\prime}$ whose associated $G$-torsor identifies with $T$, functorially in $T$ and $S$.

REMARK A.5. The displayed squares remain Cartesian in a more general topostheoretic setting; see [IZ15, 1.12, 1.16]. 


\section{Appendix B. Lifting points of non-quasi-separated algebraic stacks}

The prevalent theme of this appendix is elimination of quasi-separatedness hypotheses from several known results. This is technical but useful: for example, without our work here Proposition 4.3 would require various quasi-compactness and separatedness assumptions.

Our main goal is Theorem B.5, which extends [Knu71, II.6.4] and [LMB00, 6.3] by allowing non-quasi-compact diagonals and by replacing fields by arbitrary Henselian local rings. The proof uses the same techniques as the above references and is modeled on the proof of [LMB00, 6.3]. After discussing several consequences of Theorem B.5 (see, for instance, Corollary B.10), we combine them with Lemma B.15 (which is the backbone of Section 6) to prove in Theorems B.17 and B.18 the algebraic space analogs of the main results of [Gro68, Appendix]. The proofs in this part closely follow those of loc. cit.

B.1. $\operatorname{SEC}_{n}(\mathscr{X} / \mathscr{Y})$. Let $S$ be a scheme,

$$
\mathscr{X} \rightarrow \mathscr{Y}
$$

a representable separated morphism of $S$-algebraic stacks, and

$$
(\mathscr{X} / \mathscr{Y})^{n}:=\mathscr{X} \times_{\mathscr{Y}} \cdots \times_{\mathscr{Y}} \mathscr{X}
$$

the $n$-fold fiber product for some $n \geqslant 0$. For an $S$-scheme $S^{\prime}$, the groupoid $(\mathscr{X} / \mathscr{Y})^{n}\left(S^{\prime}\right)$ is equivalent to that of $(n+1)$-tuples $\left(s^{\prime}, x_{1}, \ldots, x_{n}\right)$, where $s^{\prime}: S^{\prime} \rightarrow \mathscr{Y}$ is an $S$-morphism and $x_{i}$ is a section of the $S$-algebraic space morphism $S^{\prime} \times_{s^{\prime}, \mathscr{Y}} \mathscr{X} \rightarrow S^{\prime}$. On $S^{\prime}$-points,

$$
(\mathscr{X} / \mathscr{Y})^{n} \rightarrow \mathscr{Y}
$$

maps $\left(s^{\prime}, x_{1}, \ldots, x_{n}\right)$ to $s^{\prime}$. Define the $S$-substack

$$
\operatorname{SEC}_{n}(\mathscr{X} / \mathscr{Y}) \subset(\mathscr{X} / \mathscr{Y})^{n}
$$

by requiring the $x_{i}$ to be disjoint; that is,

$$
S^{\prime} \times_{x_{i}, S^{\prime} \times_{s^{\prime}, \mathscr{Y}}} \mathscr{X}, x_{j} S^{\prime}=\emptyset \quad \text { for } i \neq j .
$$

By [SP, 03KP], each $x_{i}$ is a closed immersion, so the inclusion

$$
\operatorname{SEC}_{n}(\mathscr{X} / \mathscr{Y}) \subset(\mathscr{X} / \mathscr{Y})^{n}
$$

is representable by open immersions. Thus, $\operatorname{SEC}_{n}(\mathscr{X} / \mathscr{Y})$ is an algebraic stack by [SP, 06DC] (applied to the base change of a smooth atlas of $(\mathscr{X} / \mathscr{Y})^{n}$; compare also with [LMB00, 4.5(ii)]). The formation of

$$
\operatorname{SEC}_{n}(\mathscr{X} / \mathscr{Y}) \subset(\mathscr{X} / \mathscr{Y})^{n} \rightarrow \mathscr{Y}
$$

commutes with base change along any $\mathscr{Y}^{\prime} \rightarrow \mathscr{Y}$. 
B.2. $\operatorname{ET}_{n}(\mathscr{X} / \mathscr{Y})$. In the setup of Section B.1, if $\mathscr{Y}$, and hence also $\operatorname{SEC}_{n}(\mathscr{X} / \mathscr{Y})$, is an algebraic space, then the symmetric group $\mathfrak{S}_{n}$ acts freely on $\operatorname{SEC}_{n}(\mathscr{X} / \mathscr{Y})$ by permuting the $x_{i}$. Sheafification of the constant presheaf $\mathfrak{S}_{n}$ to the constant group $\left(\mathfrak{S}_{n}\right)_{S}$ retains both the action and its freeness, so the quotient sheaf

$$
\operatorname{ET}_{n}(\mathscr{X} / \mathscr{Y}):=\operatorname{SEC}_{n}(\mathscr{X} / \mathscr{Y}) /\left(\mathfrak{S}_{n}\right)_{S}
$$

is an $S$-algebraic space by [SP, 06PH] (or, in many situations, already by [LMB00, 8.1.1 and 10.6]). By descent, if $S^{\prime}$ is an $S$-scheme, then $\operatorname{ET}_{n}(\mathscr{X} / \mathscr{Y})\left(S^{\prime}\right)$ is the set of pairs $\left(s^{\prime}, x\right)$, where $s^{\prime}: S^{\prime} \rightarrow \mathscr{Y}$ is an $S$-morphism and $x \subset S^{\prime} \times_{s^{\prime}, \mathscr{Y}} \mathscr{X}$ is a closed subspace with $x \rightarrow S^{\prime}$ finite étale of degree $n$.

If $\mathscr{Y}$ is merely an algebraic stack, then we define the $S$-stack $\operatorname{ET}_{n}(\mathscr{X} / \mathscr{Y})$ by letting $\operatorname{ET}_{n}(\mathscr{X} / \mathscr{Y})\left(S^{\prime}\right)$ be the groupoid of pairs $\left(s^{\prime}, x\right)$ as in the previous sentence. The formation of

$$
\operatorname{SEC}_{n}(\mathscr{X} / \mathscr{Y}) \rightarrow \operatorname{ET}_{n}(\mathscr{X} / \mathscr{Y}) \rightarrow \mathscr{Y}
$$

commutes with base change along any $y^{\prime}: \mathscr{Y}^{\prime} \rightarrow \mathscr{Y}$. We choose $y^{\prime}$ to be a smooth cover by a scheme, and combine the previous paragraph with [SP, 05UL] (compare with [LMB00, 4.3.2]) to deduce that

(1) $\operatorname{ET}_{n}(\mathscr{X} / \mathscr{Y})$ is algebraic;

(2) $\operatorname{SEC}_{n}(\mathscr{X} / \mathscr{Y}) \rightarrow \mathrm{ET}_{n}(\mathscr{X} / \mathscr{Y})$ and $\mathrm{ET}_{n}(\mathscr{X} / \mathscr{Y}) \rightarrow \mathscr{Y}$ are representable; and

(3) $\operatorname{SEC}_{n}(\mathscr{X} / \mathscr{Y}) \rightarrow \operatorname{ET}_{n}(\mathscr{X} / \mathscr{Y})$ is finite étale of degree $n !$.

Moreover, (2)-(3) imply the claims about $\mathrm{ET}_{n}(\mathscr{X} / \mathscr{Y}) \rightarrow \mathscr{Y}$ in (4) below.

(4) If $\mathscr{X} \rightarrow \mathscr{Y}$ is smooth (respectively, étale), then so are

$$
\operatorname{SEC}_{n}(\mathscr{X} / \mathscr{Y}) \rightarrow \mathscr{Y} \quad \text { and } \operatorname{ET}_{n}(\mathscr{X} / \mathscr{Y}) \rightarrow \mathscr{Y} .
$$

LEMMA B.3. In the setup of Sections B.1-B.2, suppose that $\mathscr{X}$ is a scheme and $\mathscr{Y}$ is an algebraic space.

(a) If $\mathscr{X} \rightarrow S$ is separated and $n>0$, then the $S$-algebraic space $\operatorname{ET}_{n}(\mathscr{X} / \mathscr{Y})$ is separated.

(b) If $\mathscr{X} \rightarrow S$ is quasi-affine, $\mathscr{Y} \rightarrow S$ is quasi-separated, $\mathscr{X} \rightarrow \mathscr{Y}$ is locally of finite type, and $n>0$, then $\mathrm{ET}_{n}(\mathscr{X} / \mathscr{Y})$ is a scheme. 


\section{Proof.}

(a) By $[\mathrm{SP}, 03 \mathrm{HK}], \Delta_{\mathscr{Y} / S}$ is separated, so

$$
(\mathscr{X} / \mathscr{Y})^{n} \rightarrow S \text { along with } \operatorname{SEC}_{n}(\mathscr{X} / \mathscr{Y}) \rightarrow S
$$

inherits separatedness of $\mathscr{X} \rightarrow S$. Thus, [EGA II, 5.4.3(i)] and [EGA IV , 18.12.6] prove that

$\operatorname{SEC}_{n}(\mathscr{X} / \mathscr{Y}) \times_{S}\left(\mathfrak{S}_{n}\right)_{S} \rightarrow \operatorname{SEC}_{n}(\mathscr{X} / \mathscr{Y}) \times_{S} \operatorname{SEC}_{n}(\mathscr{X} / \mathscr{Y}), \quad(c, \sigma) \mapsto(c, c \sigma)$

is a closed immersion, and the separatedness of $\operatorname{SEC}_{n}(\mathscr{X} / \mathscr{Y}) /\left(\mathfrak{S}_{n}\right)_{S} \rightarrow S$ follows.

(b) By [SP, 03HK] and [EGA $\left.\mathbf{I V}_{4}, 18.12 .12\right], \Delta \mathscr{Y} / S$ is quasi-affine, so $(\mathscr{X} / \mathscr{Y})^{n} \rightarrow S$ inherits quasi-affineness from $\mathscr{X} \rightarrow S$. Once the open immersion

$$
\operatorname{SEC}_{n}(\mathscr{X} / \mathscr{Y}) \hookrightarrow(\mathscr{X} / \mathscr{Y})^{n}
$$

is proved to be quasi-compact and hence also quasi-affine,

$$
\operatorname{SEC}_{n}(\mathscr{X} / \mathscr{Y}) \rightarrow S
$$

will have to be quasi-affine, too, and [SP, 07S7] (or [SGA $3_{\text {I new }}, 4.1$ ]) will give the claim.

The open $\operatorname{SEC}_{n}(\mathscr{X} / \mathscr{Y}) \subset(\mathscr{X} / \mathscr{Y})^{n}$ is the complement of the union of the diagonals

$$
\Delta_{i, j}:(\mathscr{X} / \mathscr{Y})^{n-1} \hookrightarrow(\mathscr{X} / \mathscr{Y})^{n} \quad \text { for } i \neq j .
$$

Each $\Delta_{i, j}$ is a closed immersion that is of finite presentation by [EGA IV $\mathbf{V}_{\mathbf{1}}$, $1.4 .3(\mathrm{v})]$. The inclusion of the complement of $\Delta_{i, j}$ is therefore a quasicompact open immersion, and hence so is

$$
\operatorname{SEC}_{n}(\mathscr{X} / \mathscr{Y}) \hookrightarrow(\mathscr{X} / \mathscr{Y})^{n} .
$$

B.4. $\mathrm{P}_{n}(\mathscr{X} / \mathscr{Y})$. In the setup of Sections B.1-B.2, suppose that $n>0$ and $\mathscr{X}$ is an algebraic space. As in Section B.2, $\left(\mathfrak{S}_{n}\right)_{S}$ acts (possibly nonfreely) on the algebraic space $\operatorname{SEC}_{n}(\mathscr{X} / \mathscr{Y})$. We fix a closed embedding $\iota:\left(\mathfrak{S}_{n}\right)_{S} \hookrightarrow\left(\mathrm{GL}_{n}\right)_{S}$, for example, one furnished by permutation matrices, and set

$$
\mathrm{P}_{n}(\mathscr{X} / \mathscr{Y}):=\left(\operatorname{SEC}_{n}(\mathscr{X} / \mathscr{Y}) \times_{S}\left(\mathrm{GL}_{n}\right)_{S}\right) /\left(\mathfrak{S}_{n}\right)_{S},
$$

where $\left(\mathfrak{S}_{n}\right)_{S}$ acts by

$$
((c, g), \sigma) \mapsto\left(c \sigma, \iota(\sigma)^{-1} g\right)
$$


By [SP, 06PH] (or, in many situations, already by [LMB00, 8.1.1 and 10.6]), $\mathrm{P}_{n}(\mathscr{X} / \mathscr{Y})$ is an $S$-algebraic space (that depends on $\left.\iota\right)$. The formation of

$$
\mathrm{P}_{n}(\mathscr{X} / \mathscr{Y}) \rightarrow \operatorname{ET}_{n}(\mathscr{X} / \mathscr{Y}) \rightarrow \mathscr{Y}
$$

commutes with base change along any representable $y^{\prime}: \mathscr{Y}^{\prime} \rightarrow \mathscr{Y}$. Moreover, Section B.2 proves the following.

(1) If $\mathscr{Y}$ is an algebraic space, then $\mathrm{P}_{n}(\mathscr{X} / \mathscr{Y})$ is a $\mathrm{GL}_{n}$-torsor over $\operatorname{ET}_{n}(\mathscr{X} / \mathscr{Y})$.

We choose $y^{\prime}$ to be a smooth surjection from an algebraic space and use (1) to obtain the following.

(2) $\mathrm{P}_{n}(\mathscr{X} / \mathscr{Y}) \rightarrow \operatorname{ET}_{n}(\mathscr{X} / \mathscr{Y})$ is smooth.

We are now ready for the main result of this appendix.

THEOREM B.5. Let $S$ be a scheme, $\mathscr{Z}$ an $S$-algebraic stack, and $\operatorname{Spec} R$ an $S$ scheme with $R$ Henselian local. If the $S$-fiber of $\Delta_{\mathscr{Z} / S}$ over the image of the closed point of $\operatorname{Spec} R$ is separated (in particular, if $\mathscr{Z}$ is an algebraic space), then for every $z \in \mathscr{Z}(R)$ there is a smooth (respectively, étale if $\mathscr{Z}$ is an algebraic space) morphism

$$
Z \rightarrow \mathscr{Z}
$$

with $Z$ an affine scheme such that $z$ lifts to $Z(R)$.

See Remark B.6 for an example of how the $S$-fibral hypothesis on $\Delta_{\mathscr{Z} / S}$ is useful practice.

Proof of Theorem B.5 in the case when $\Delta_{\mathscr{Z} / S}$ is separated and $R$ is a field. We use [SP, 04X5] (or [Con07a, 1.3]) to change $S$ to Spec $\mathbb{Z}$. (We later use the gained affineness of $S$ to ensure the quasi-affineness of $W \rightarrow S$, which we need for Lemma B.3(b). Quasi-separatedness of $S$ would suffice (such quasi-separatedness is an omnipresent convention in [LMB00]).) The new diagonal $\Delta_{\mathscr{Z} / \mathbb{Z}}$ is separated, being the composite

$$
\mathscr{Z} \stackrel{\Delta_{\mathscr{Z} / S}}{\longrightarrow} \mathscr{Z} \times_{S} \mathscr{Z} \rightarrow \mathscr{Z} \times_{\mathbb{Z}} \mathscr{Z}
$$

in which the second map inherits separatedness from $\Delta_{S / \mathbb{Z}}$.

We choose a smooth (respectively, étale if $\mathscr{Z}$ is an algebraic space) surjection

$$
W \rightarrow \mathscr{Z}
$$


with $W$ a separated $S$-scheme, and set

$$
W_{R}:=W \times_{\mathscr{Z}, z} \operatorname{Spec} R \text {. }
$$

Since $W \rightarrow S$ and $\Delta_{\mathscr{Z} / S}$ are separated, so is $W \rightarrow \mathscr{Z}$. Thus, $W_{R}$ is a separated smooth nonempty $R$-algebraic space. We retain these properties of $W_{R}$ as well as smoothness (respectively, étaleness if $\mathscr{Z}$ is an algebraic space) but not surjectivity of $W \rightarrow \mathscr{Z}$ by replacing $W$ by a suitable affine open $W^{\prime} \subset W$ and $W_{R}$ by

$$
W^{\prime} \times{ }_{W} W_{R}
$$

this gains affineness of $W \rightarrow S$.

We apply [EGA $I_{4}, 17.16 .3$ (ii)] to an étale cover of $W_{R}$ by a scheme to find a finite separable field extension $R^{\prime} / R$ and a $w \in W_{R}\left(R^{\prime}\right)$. We set $n:=\left[R^{\prime}: R\right]$, and we use [Knu71, II.6.2] to assume that $\operatorname{Spec} R^{\prime} \stackrel{w}{\rightarrow} W_{R}$ is a monomorphism, and hence even a closed immersion by [SP, 04NX and 05W8] (alternatively, by [LMB00, A.2] and [EGA IV $\left.\left.\mathbf{I V}_{4}, 18.12 .6\right]\right)$. Then, by Section B.2,

$$
w \in \operatorname{ET}_{n}\left(W_{R} / R\right)(R) .
$$

Moreover, $w$ lifts to a $p \in \mathrm{P}_{n}\left(W_{R} / R\right)(R)$ thanks to the triviality of the $\left(\mathrm{GL}_{n}\right)_{R^{-}}$ torsor

$$
\mathrm{P}_{n}\left(W_{R} / R\right) \times_{\mathrm{ET}_{n}\left(W_{R} / R\right), w} \operatorname{Spec} R .
$$

The images of $w$ in $\operatorname{ET}_{n}(W / \mathscr{Z})(R)$ and of $p$ in $\mathrm{P}_{n}(W / \mathscr{Z})(R)$ lift $z$. As observed in Section B.2(4) and Section B.4(2), $\mathrm{P}_{n}(W / \mathscr{Z})$ inherits $\mathscr{Z}$-smoothness from $W$, and $\mathrm{ET}_{n}(W / \mathscr{Z})$ also inherits $\mathscr{Z}$-étaleness if $\mathscr{Z}$ is an algebraic space.

We use the argument above to pass to $\mathrm{P}_{n}(W / \mathscr{Z})$, and hence to reduce to the case when $\mathscr{Z}$ is an algebraic space. Then we use the same argument and Lemma B.3(a) to pass to $\operatorname{ET}_{n}(W / \mathscr{Z})$ and to assume further that $\mathscr{Z}$ is separated. We repeat using Lemma B.3(b) instead to assume that $\mathscr{Z}$ is even a scheme, in which case an open affine $Z \subset \mathscr{Z}$ through which $z$ factors suffices.

Proof of Theorem B.5 in the case when $R$ is a field. If $\mathscr{Z}$ is an algebraic space, then $\Delta_{\mathscr{Z} / S}$ is separated as before. If not, then let

$$
W \rightarrow \mathscr{Z}
$$

be a smooth surjection with $W$ a separated $S$-scheme. For $n \geqslant 1$, the $n$-fold fiber product $(W / \mathscr{Z})^{n}$ is a smooth $\mathscr{Z}$-algebraic space with a $\left(\mathfrak{S}_{n}\right)_{S}$-action. By [SP, 04TK and 04X0] (see also [LMB00, 4.3.1]), the quotient stack

$$
(W / \mathscr{Z})^{n} /\left(\mathfrak{S}_{n}\right)_{S}
$$


is algebraic, and

$$
(W / \mathscr{Z})^{n} \rightarrow(W / \mathscr{Z})^{n} /\left(\mathfrak{S}_{n}\right)_{S}
$$

is a smooth surjection. Therefore, $(W / \mathscr{Z})^{n} /\left(\mathfrak{S}_{n}\right)_{S}$ inherits $\mathscr{Z}$-smoothness from $(W / \mathscr{Z})^{n}$.

Claim B.5.1. The diagonal of $(W / \mathscr{Z})^{n} /\left(\mathfrak{S}_{n}\right)_{S} \rightarrow S$ is separated.

Proof. We need to prove that

$$
a:(W / \mathscr{Z})^{n} \times_{S}\left(\mathfrak{S}_{n}\right)_{S} \rightarrow(W / \mathscr{Z})^{n} \times_{S}(W / \mathscr{Z})^{n}, \quad(x, \sigma) \mapsto(x, x \sigma)
$$

is separated. The diagonal $\Delta_{\mathrm{pr}_{1} \circ a}$ of the composition of $a$ and

$$
(W / \mathscr{Z})^{n} \times_{S}(W / \mathscr{Z})^{n} \stackrel{\mathrm{pr}_{1}}{\rightarrow}(W / \mathscr{Z})^{n}
$$

is

$$
(W / \mathscr{Z})^{n} \times_{S}\left(\mathfrak{S}_{n}\right)_{S} \stackrel{\Delta_{a}}{\rightarrow} F \stackrel{f}{\rightarrow}(W / \mathscr{Z})^{n} \times_{S}\left(\mathfrak{S}_{n}\right)_{S} \times_{S}\left(\mathfrak{S}_{n}\right)_{S},
$$

where $f$ is a base change of $\Delta_{\mathrm{pr}_{1}}$. Since

$$
\Delta_{\mathrm{pr}_{1} \circ a}=\operatorname{id}_{(W / \mathscr{Z})^{n}} \times \Delta_{\left(\mathfrak{S}_{n}\right)_{S} / S}
$$

and $\Delta_{\mathrm{pr}_{1}}$ is separated, $\Delta_{a}$ is a closed immersion.

To reduce to the previous case, it therefore suffices to lift $z$ to $\left((W / \mathscr{Z})^{n} /\left(\mathfrak{S}_{n}\right)_{S}\right)(R)$ for some $n \geqslant 1$. For this, since the formation of

$$
(W / \mathscr{Z})^{n} \rightarrow(W / \mathscr{Z})^{n} /\left(\mathfrak{S}_{n}\right)_{S} \rightarrow \mathscr{Z}
$$

commutes with base change along any $S^{\prime} \rightarrow S$, we choose $S^{\prime}$ to be the point of $S$ below $z$ to reduce to the case when $\Delta_{\mathscr{Z} / S}$ is separated. The proof of the previous case then lifts $z$ to $\operatorname{ET}_{n}(W / \mathscr{Z})(R)$ for some $n \geqslant 1$, and the presence of a $\mathscr{Z}$ morphism

$$
\operatorname{ET}_{n}(W / \mathscr{Z}) \rightarrow(W / \mathscr{Z})^{n} /\left(\mathfrak{S}_{n}\right)_{S}
$$

obtained from the construction finishes the proof.

Proof of Theorem B.5 in the general case. The result follows by combining the proved field case with the following lemma.

LEMMA B.5.2. Let $S$ be a scheme, $\mathscr{Z}$ an $S$-algebraic stack, and $\operatorname{Spec} R$ an $S$ scheme with $(R, \mathfrak{m})$ Henselian local. For a smooth $S$-morphism

$$
Z \rightarrow \mathscr{Z}
$$

with $Z$ an algebraic space, if the pullback $z_{0} \in \mathscr{Z}(R / \mathfrak{m})$ of a $z \in \mathscr{Z}(R)$ lifts to a $\widetilde{z}_{0} \in Z(R / \mathfrak{m})$, then $z$ lifts to $a \tilde{z} \in Z(R)$ that pulls back to $\widetilde{z}_{0}$. 
Proof. We set

$$
Z_{R}:=Z \times \times_{\mathscr{Z}, z} \operatorname{Spec} R,
$$

so $\widetilde{z}_{0}$ gives rise to an element $\widetilde{z}_{0} \in Z_{R}(R / \mathfrak{m})$. To lift the latter to a desired section of

$$
Z_{R} \rightarrow \operatorname{Spec} R
$$

we use the established field case of Theorem B.5 to replace $Z_{R}$ by an étale $Z_{R^{-}}$ scheme to which $\widetilde{z}_{0}$ lifts and then apply [EGA $\left.\mathbf{I V}_{4}, 18.5 .17\right]$.

REMARK B.6. We do not know if the $S$-fibral separatedness assumption on $\Delta_{\mathscr{Z} / S}$ is necessary in Theorem B.5. Due to separatedness of group schemes over fields and Lemma A.2(b), this assumption is met if $\mathscr{Z}=\mathbf{B} G$ for a flat and locally of finite presentation $S$-group algebraic space $G$ whose $S$-fiber over the image of the closed point of Spec $R$ is a scheme.

COROLlary B.7. Let $S$ be a scheme, $\operatorname{Spec} R$ an $S$-scheme with $(R, \mathfrak{m})$ Henselian local, and $\mathscr{Z}$ an $S$-algebraic stack such that $\Delta_{\mathscr{Z} / S}$ has a separated $S$ fiber over the image of the closed point of $\mathrm{Spec} R$. There is a separated $S$-scheme $Z$ and a smooth (respectively, étale if $\mathscr{Z}$ is an algebraic space) $Z \rightarrow \mathscr{Z}$ for which

$$
Z(R / \mathfrak{a}) \rightarrow \mathscr{Z}(R / \mathfrak{a})
$$

is essentially surjective for every ideal $\mathfrak{a} \subset R$; if the number of isomorphism classes of objects of $\mathscr{Z}(R / \mathfrak{m})$ is finite, then $Z$ can be taken to be affine.

Proof. We use Theorem B.5 to build a $Z$ to which every $z \in \mathscr{Z}(R / \mathfrak{m})$ lifts, and apply Lemma B.5.2.

COROLlaRY B.8. For a Henselian local ring $R$ and a smooth $R$-algebraic stack $\mathscr{Z}$ such that $\Delta_{\mathscr{Z} / R}$ has a separated $R$-fiber over the closed point of $\operatorname{Spec} R$, the pullback map

$$
\mathscr{Z}(R) \rightarrow \mathscr{Z}(R / \mathfrak{a})
$$

is essentially surjective for every ideal $\mathfrak{a} \subsetneq R$.

Proof. Corollary B.7 reduces to the scheme case, which is a known variant of [EGA IV $4,18.5 .17]$.

We illustrate Corollary B.8 with two special cases recorded in Corollaries B.9 and B.10. 
Corollary B.9. For a Henselian local ring $R$ and an ideal $\mathfrak{a} \subset R$, every principally polarized abelian scheme over $R / \mathfrak{a}$ arises as the base change of a principally polarized abelian scheme over $R$.

Proof. By [FC90, (i) on page 95], for every $g \geqslant 0$ the moduli stack $\mathcal{A}_{g}$ of principally polarized abelian schemes of relative dimension $g$ is separated and smooth over $\mathbb{Z}$, so Corollary B.8 applies to it.

The results of B.10-B.13 play a role in the proof of Proposition 3.5.

COROllary B.10. For a Henselian local ring $R$, an ideal $\mathfrak{a} \subset R$, and a flat, locally of finite presentation $R$-group algebraic space $G$ whose special fiber is a scheme,

$$
H^{1}(R, G) \rightarrow H^{1}(R / \mathfrak{a}, G)
$$

is surjective.

Proof. By Proposition A.3 and Remark B.6, Corollary B.8 applies to BG.

\section{Remarks.}

B.11. For $\mathfrak{a} \subsetneq R$ as in Corollary B. 10 and $G$ a smooth $R$-group algebraic space,

$$
H^{1}(R, G) \rightarrow H^{1}(R / \mathfrak{a}, G)
$$

is injective: fix $G$-torsors $T$ and $T^{\prime}$, and apply Corollary B.8 to the fppf sheaf

$$
\operatorname{Isom}_{G}\left(T, T^{\prime}\right): S \mapsto\left\{G_{S^{-}} \text {torsor isomorphisms } T_{S} \stackrel{\sim}{\longrightarrow} T_{S}^{\prime}\right\},
$$

which is a torsor under the inner form $\operatorname{Aut}_{G} T$ of $G$, and hence also a smooth $R$-algebraic space. Thus, if the special fiber of $G$ is a scheme, then $H^{1}(R, G) \rightarrow H^{1}(R / \mathfrak{a}, G)$ is bijective (compare with [SGA $3_{\text {III new }}, \mathrm{XXIV,}$ 8.1(iii)]). Likewise if $G$ is commutative; see Theorem B.18.

B.12. See [Toë11, 3.4] for an analog of Corollary B.10 with $R$ Henselian local excellent, $\mathfrak{a}$ the maximal ideal, and $G$ commutative, flat, and locally of finite presentation (but with arbitrary special fiber). Under these assumptions, loc. cit. also shows the bijectivity of the analogous pullback for $H^{n}$ with $n>1$. The excellence assumption can be removed in many cases: 
Proposition B.13. For a Henselian local ring $(R, \mathfrak{m})$, an ideal $\mathfrak{a} \subset \mathfrak{m}$, and $a$ commutative flat $R$-group algebraic space $G$ of finite presentation,

$$
H^{1}(R, G) \rightarrow H^{1}(R / \mathfrak{m}, G)
$$

is surjective and

$$
H^{n}(R, G) \rightarrow H^{n}(R / \mathfrak{a}, G)
$$

is bijective for $n \geqslant 2$.

Proof. We may assume that $\mathfrak{a}=\mathfrak{m}$. We use $\left[\mathbf{E G A} \mathbf{I V}_{4}, 18.6 .14(\mathrm{ii})\right]$ to express $(R, \mathfrak{m})$ as a filtered direct limit of Henselian local rings $\left(R_{i}, \mathfrak{m}_{i}\right)$ each of which is a Henselization of a finite type $\mathbb{Z}$-algebra. By [EGA IV $\mathbf{I V}_{2}, 7.8 .3$ ] and [EGA $\mathbf{I V}_{4}$, 18.7.6], each $R_{i}$ is excellent. By [SP, 07SK and 08K0], there is an $i$ for which $G$ descends to a commutative flat $R_{i}$-group algebraic space $G_{i}$ of finite presentation.

For each $j \geqslant i$, we set $G_{j}:=\left(G_{i}\right)_{R_{j}}$. By the limit formalism for fppf cohomology, namely, by the analog of [SGA $4_{\text {II }}$, VII, 5.9],

$$
H^{n}(R, G)=\underset{j}{\lim _{\vec{j}}} H^{n}\left(R_{j}, G_{j}\right) \quad \text { and } \quad H^{n}(R / \mathfrak{m}, G)=\underset{j}{\lim } H^{n}\left(R_{j} / \mathfrak{m}_{j}, G_{j}\right) .
$$

The claims therefore reduce to the case of an excellent $R$, which is the subject of [Toë11, 3.4].

We now turn to the algebraic space analogs of the results of [Gro68, Appendix]. The proofs are analogous, too; we have decided to include them here because Lemma B.15 is of major importance for Section 6, while the rest are quick consequences.

LEMMA B.14. Let $S$ be a scheme, $j: T^{\prime} \hookrightarrow T$ a square-zero closed immersion of $S$-algebraic spaces, $G$ a commutative smooth $T$-group algebraic space, and $N$ the fppf sheaf $\operatorname{Ker}\left(G \rightarrow j_{*}\left(G_{T^{\prime}}\right)\right)$. There is a quasi-coherent sheaf $\mathcal{F}$ on $T$ such that

$$
N(\widetilde{T})=\Gamma\left(\mathcal{O}_{\widetilde{T}},\left.\mathcal{F}\right|_{\widetilde{T}}\right)
$$

for every flat $T$-algebraic space $\widetilde{T}$.

Proof. Let $\mathcal{C}_{T^{\prime} / T}$ be the conormal sheaf of $j$ (see [SP, Definition $\left.04 \mathrm{CN}\right]$ ), and let $e: T \rightarrow G$ be the unit section. Set

$$
\mathcal{F}:=j_{*} \mathcal{H o m}_{\mathcal{O}_{T^{\prime}}}\left(j^{*} e^{*} \Omega_{G / T}, \mathcal{C}_{T^{\prime} / T}\right) .
$$

By [SP, 04CQ], the formation of $\mathcal{C}_{T^{\prime} / T}$ commutes with base change along a flat $\widetilde{T} \rightarrow T$, so [SP, 061C and 061D] give the claim. 
LEMmA B.15. Let $S$ be a scheme, $T_{0} \rightarrow T$ a finite locally free morphism of $S$ algebraic spaces, and $G$ a commutative smooth $T$-group algebraic space. For $n \geqslant 0$, set

$$
C^{n}:=\operatorname{Res}_{T_{n} / T}\left(G_{T_{n}}\right),
$$

where $T_{n}=T_{0} \times_{T} \cdots \times_{T} T_{0}$ with $n+1$ factors of $T_{0}$, define the usual coboundary map $d^{n}: C^{n} \rightarrow C^{n+1}$, and set $Z^{n}:=\operatorname{Ker} d^{n}$. The induced map

$$
d^{n}: C^{n} \rightarrow Z^{n+1}
$$

is a smooth morphism of $T$-group algebraic spaces.

Proof. By [SP, 05YF and 04AK] (see also [Ols06, 1.5]), $C^{n}$ and $Z^{n+1}$ are locally of finite presentation $T$-group algebraic spaces. By [SP, 04AM] (see also [LMB00, 4.15(ii)]), it remains to argue that

$$
d^{n}: C^{n} \rightarrow Z^{n+1}
$$

is formally smooth. For this, we base change to assume that $T$ is affine,

$$
j: T^{\prime} \hookrightarrow T
$$

is a closed subscheme defined by a square-zero ideal, and $a^{\prime} \in C^{n}\left(T^{\prime}\right)$ is such that

$$
b^{\prime}:=d^{n}\left(a^{\prime}\right) \in Z^{n+1}\left(T^{\prime}\right)
$$

lifts to a $b \in Z^{n+1}(T)$.

We need to lift $a^{\prime}$ to an $a \in C^{n}(T)$ subject to $d^{n}(a)=b$. Formal smoothness of $C^{n}$ inherited from $G$ lifts $a^{\prime}$ to an $\widetilde{a} \in C^{n}(T)$. We replace $b$ by $b-d^{n}(\widetilde{a})$ to reduce to the case when $a^{\prime}=b^{\prime}=0$. We set

$$
N:=\operatorname{Ker}\left(G \rightarrow j_{*}\left(G_{T^{\prime}}\right)\right),
$$

so the cocycle $b$ is valued in the subsheaf $N \subset G$.

By Lemma B.14,

$$
H^{n+1}\left(T_{0} / T, N\right)=H^{n+1}\left(T_{0} / T, \mathcal{F}\right)
$$

for some quasi-coherent sheaf $\mathcal{F}$ on $T_{\text {fppf }}$, so $H^{n+1}\left(T_{0} / T, N\right)=0$ by [Gro59, B, Lemme 1.1]. The existence of an $N$-valued cochain $a$ with $d^{n}(a)=b$ follows.

Lemma B.16. Let $R$ be a Henselian local ring, $\mathfrak{a} \subsetneq R$ an ideal, $G$ a commutative smooth $R$-group algebraic space, $R_{0}$ an $R$-algebra that is finite free as an $R$ module, $T_{0}=\operatorname{Spec} R_{0}$, and $T=\operatorname{Spec} R$. 
(a) If $R$ is strictly Henselian and $n \geqslant 1$, then $H^{n}\left(T_{0} / T, G\right)=0$.

(b) The map

$$
H^{n}\left(T_{0} / T, G\right) \rightarrow H^{n}\left(\left(T_{0}\right)_{R / \mathfrak{a}} / T_{R / \mathfrak{a}}, G\right)
$$

is surjective for $n \geqslant 0$ and bijective for $n \geqslant 1$.

Proof. For an $x \in Z^{n}(R)$ with $n \geqslant 1$, we set

$$
Z_{x}^{n}:=\operatorname{Spec} R \times_{x, Z^{n}, d^{n-1}} C^{n-1} \quad\left(\text { so } Z_{0}^{n}=Z^{n-1}\right) .
$$

By Lemma B.15, $Z_{x}^{n}$ is $R$-smooth, so [EGA IV $\mathbf{I V}_{4}, 17.16 .3$ (ii)] proves (a) by showing that $Z_{x}^{n}(R) \neq \emptyset$. Also, Corollary B.8 applied to $Z_{x}^{n}$ (respectively, $Z_{0}^{n}$ ) proves the injectivity (respectively, surjectivity) in (b).

THEOREM B.17. Let $S$ be a scheme and $f: S_{f p p f} \rightarrow S_{\text {ét }}$ the canonical morphism of sites. For a commutative smooth $S$-group algebraic space $G$, one has

$$
\mathbf{R}^{n} f_{*} G=0 \text { for every } n \geqslant 1 \text {; }
$$

in particular,

$$
H_{e t}^{n}(S, G)=H_{f p p f}^{n}(S, G)
$$

for such $G$ and $n$.

Proof. By [Gro68, 11.1], it suffices to prove that, if $S=\operatorname{Spec} R$ with $R$ strictly Henselian local, then $H_{\mathrm{fppf}}^{n}(S, G)=0$ for $n \geqslant 1$. For this, we combine [Gro68, 11.2] with Lemma B.16(a).

THEOREM B.18. Let $R$ be a ring, $\mathfrak{a} \subsetneq R$ an ideal, and $G$ a commutative smooth $R$-group algebraic space. If $\mathfrak{a}$ is nilpotent or if $R$ is Henselian local, then the pullback map

$$
H^{n}(R, G) \rightarrow H^{n}(R / \mathfrak{a}, G)
$$

is bijective for $n \geqslant 1$.

Proof. For $j: \operatorname{Spec} R / \mathfrak{a} \hookrightarrow \operatorname{Spec} R$, set $N:=\operatorname{Ker}\left(G \rightarrow j_{*}\left(G_{R / \mathfrak{a}}\right)\right)$. Thanks to Corollary B.8,

$$
0 \rightarrow N \rightarrow G \rightarrow j_{*}\left(G_{R / \mathfrak{a}}\right) \rightarrow 0
$$

is short exact in $(\operatorname{Spec} R)_{\text {ét }}$. Thus, Theorem B.17 reduces us to proving that

$$
H_{\mathrm{et}}^{n}(R, N)=0 \quad \text { for } n \geqslant 1
$$


The case when $\mathfrak{a}$ is nilpotent reduces to the case when $\mathfrak{a}^{2}=0$, in which

$$
H_{\mathrm{et}}^{n}(R, N)=0 \quad \text { for } n \geqslant 1
$$

by Lemma B.14 combined with the vanishing of quasi-coherent cohomology of affine schemes.

In the case when $R$ is Henselian local, the analog of [Gro68, 11.2] for étale cohomology and finite étale covers reduces us to proving that

$$
H^{n}\left(R_{0} / R, N\right)=0 \text { for } n \geqslant 1
$$

and an étale $R$-algebra $R_{0}$ that is finite as an $R$-module. We set $T_{0}:=\operatorname{Spec} R_{0}$ and $T:=\operatorname{Spec} R$, consider sheaves of cochains for $T_{0} / T$ as in Lemma B.15, decorate them with subscripts to indicate the relevant groups, and note that by Corollary B.8

$$
0 \rightarrow C_{N}^{n}(R) \rightarrow C_{G}^{n}(R) \rightarrow C_{j_{*}\left(G_{R / \mathfrak{a}}\right)}^{n}(R) \rightarrow 0
$$

is short exact for $n \geqslant 0$. The resulting short exact sequence of complexes gives the exact sequence

$$
\begin{aligned}
\cdots & \rightarrow H^{n}\left(\left(T_{0}\right)_{R / \mathfrak{a}} / T_{R / \mathfrak{a}}, G\right) \rightarrow H^{n+1}\left(T_{0} / T, N\right) \\
& \rightarrow H^{n+1}\left(T_{0} / T, G\right) \rightarrow H^{n+1}\left(\left(T_{0}\right)_{R / \mathfrak{a}} / T_{R / \mathfrak{a}}, G\right) \rightarrow \cdots,
\end{aligned}
$$

so the desired $H^{n}\left(T_{0} / T, N\right)=0$ for $n \geqslant 1$ follows from Lemma B.16(b).

\section{References}

[Art69] M. Artin, 'Algebraization of formal moduli. I', in Global Analysis (Papers in Honor of K. Kodaira) (University of Tokyo Press, Tokyo, 1969), 21-71; MR 0260746 (41 \#5369).

[BLR90] S. Bosch, W. Lütkebohmert and M. Raynaud, Néron Models, Ergebnisse der Mathematik und ihrer Grenzgebiete (3) [Results in Mathematics and Related Areas (3)], 21 (Springer, Berlin, 1990); MR 1045822 (91i:14034).

[BouA] N. Bourbaki, Éléments de mathématique. Algèbre (chap. I-III, Hermann (1970); chap. IV-VII, Masson (1981); chap. VIII-X, Springer (2007, 2012) (in French)).

[BT14] D. P. Băć and N. Q. Thăńg, 'On the topology on group cohomology of algebraic groups over complete valued fields', J. Algebra 399 (2014), 561-580; doi:10.1016/j.jalgebra.2013.08.041; MR 3144603.

[CGP10] B. Conrad, O. Gabber and G. Prasad, Pseudo-Reductive Groups, New Mathematical Monographs, 17 (Cambridge University Press, Cambridge, 2010); MR 2723571 (2011k:20093).

[CLO12] B. Conrad, M. Lieblich and M. Olsson, 'Nagata compactification for algebraic spaces', J. Inst. Math. Jussieu 11(4) (2012), 747-814; doi:10.1017/S1474748011 000223 ; MR 2979821. 
[Con07a] B. Conrad, 'Arithmetic moduli of generalized elliptic curves', J. Inst. Math. Jussieu 6(2) (2007), 209-278; doi:10.1017/S1474748006000089; MR 2311664 (2008e:11073).

[Con07b] B. Conrad, 'Deligne's notes on Nagata compactifications', J. Ramanujan Math. Soc. 22(3) (2007), 205-257; MR 2356346 (2009d:14002).

[Con12] B. Conrad, 'Weil and Grothendieck approaches to adelic points', Enseign. Math. (2) 58(1-2) (2012), 61-97; MR 2985010.

[EGA II] A. Grothendieck and J. Dieudonné, 'Éléments de géométrie algébrique. II. Étude globale élémentaire de quelques classes de morphismes', Publ. Math. Inst. Hautes Études Sci. (8) (1961), 222 (in French); MR 0163909 (29\#1208).

[EGA IV $\left.{ }_{1}\right]$ A. Grothendieck and J. Dieudonné, 'Éléments de géométrie algébrique. IV. Étude locale des schémas et des morphismes de schémas. I', Publ. Math. Inst. Hautes Études Sci. (20) (1964), 259 (in French); MR 0173675 (30 \#3885).

[EGA IV $\mathrm{IV}_{2}$ A. Grothendieck and J. Dieudonné, 'Éléments de géométrie algébrique. IV. Étude locale des schémas et des morphismes de schémas. II', Publ. Math. Inst. Hautes Études Sci. (24) (1965), 231 (in French); MR 0199181 (33 \#7330).

[EGA IV $\left.\mathrm{I}_{3}\right]$ A. Grothendieck and J. Dieudonné, 'Éléments de géométrie algébrique. IV. Étude locale des schémas et des morphismes de schémas. III', Publ. Math. Inst. Hautes Études Sci. (28) (1966), 255; MR 0217086 (36 \#178).

$\left[\mathrm{EGA} \mathrm{IV}_{4}\right] \quad$ A. Grothendieck and J. Dieudonné, 'Éléments de géométrie algébrique. IV. Étude locale des schémas et des morphismes de schémas IV', Publ. Math. Inst. Hautes Études Sci. (32) (1967), 361 (in French); MR 0238860 (39 \#220).

[FC90] G. Faltings and C.-L. Chai, Degeneration of Abelian Varieties, Ergebnisse der Mathematik und ihrer Grenzgebiete (3) [Results in Mathematics and Related Areas (3)], 22 (Springer, Berlin, 1990). With an appendix by David Mumford; MR 10833 53 (92d:14036).

[Gir71] J. Giraud, Cohomologie non abélienne, Die Grundlehren der mathematischen Wissenschaften, Band 179 (Springer, Berlin, 1971) (in French); MR 0344253 (49 \#8992).

[GGMB14] O. Gabber, P. Gille and L. Moret-Bailly, 'Fibrés principaux sur les corps valués henséliens', Algebr. Geom. 1(5) (2014), 573-612 (French, with English and French summaries); MR 3296806.

[Gro59] A. Grothendieck, Technique de descente et théorèmes d'existence en géometrie algébrique. I. Généralités. Descente par morphismes fidèlement plats, Séminaire Bourbaki, Exp. No. 190, Vol. 5 (Société Mathématique de France, Paris, 1995), 299-327. (in French); MR 1603475.

[Gro68] A. Grothendieck, 'Le groupe de Brauer. III. Exemples et compléments', in Dix Exposés sur la Cohomologie des Schémas (North-Holland, Amsterdam, 1968), 88188 (in French); MR 0244271 (39 \#5586c).

[IZ15] L. Illusie and W. Zheng, 'Quotient stacks and equivariant étale cohomology algebras: Quillen's theory revisited', J. Algebraic Geom. to appear, (2015), available at arXiv:abs/1305.0365.

[Knu71] D. Knutson, Algebraic Spaces, Lecture Notes in Mathematics, 203 (Springer, Berlin, 1971); MR 0302647 (46 \#1791).

[LMB00] G. Laumon and L. Moret-Bailly, Champs Algébriques, Ergebnisse der Mathematik und ihrer Grenzgebiete. 3. Folge. A Series of Modern Surveys in Mathematics [Results in Mathematics and Related Areas. 3rd Series. A Series of Modern Surveys in Mathematics], 39 (Springer, Berlin, 2000) (in French);

MR 1771927 (2001f:14006). 
[MB01] L. Moret-Bailly, 'Problèmes de Skolem sur les champs algébriques', Compositio Math. 125(1) (2001), 1-30 (French, with English summary); MR 1818054 (2002c:11067); doi:10.1023/A:1002686625404.

[MB12] L. Moret-Bailly, 'Un théorème de l'application ouverte sur les corps valués algébriquement clos', Math. Scand. 111(2) (2012), 161-168 (French, with English and French summaries); MR 3023520.

[Mil06] J. S. Milne, Arithmetic Duality Theorems, 2nd edn (BookSurge, LLC, Charleston, SC, 2006); MR 2261462 (2007e:14029).

[Ols06] M. C. Olsson, 'Hom-stacks and restriction of scalars', Duke Math. J. 134(1) (2006), 139-164; doi:10.1215/S0012-7094-06-13414-2; MR 2239345 (2007f:14002).

[Ser02] J.-P. Serre, Galois Cohomology, Springer Monographs in Mathematics, Corrected reprint of the 1997 English edition (Springer, Berlin, 2002) Translated from the French by Patrick Ion and revised by the author; MR 1867431 (2002i:12004).

[SGA 3 $3_{\text {Inew }}$ ] P. Gille and P. Polo (Eds.), Schémas en groupes (SGA 3). Tome I. Propriétés générales des schémas en groupes, Documents Mathématiques (Paris) [Mathematical Documents (Paris)], 7 (Société Mathématique de France, Paris, 2011) (in French). Séminaire de Géométrie Algébrique du Bois Marie 1962-64. [Algebraic Geometry Seminar of Bois Marie 1962-64]; A seminar directed by M. Demazure and A. Grothendieck with the collaboration of M. Artin, J.-E. Bertin, P. Gabriel, M. Raynaud and J.-P. Serre; Revised and annotated edition of the 1970 French original; MR 2867621.

[SGA $3_{\text {III new }}$ P. Gille and P. Polo (Eds.), Schémas en groupes (SGA 3). Tome III. Structure des schémas en groupes réductifs, Documents Mathématiques (Paris) [Mathematical Documents (Paris)], 8 (Société Mathématique de France, Paris, 2011) (in French). Séminaire de Géométrie Algébrique du Bois Marie 1962-64. [Algebraic Geometry Seminar of Bois Marie 1962-64]; A seminar directed by M. Demazure and A. Grothendieck with the collaboration of M. Artin, J.-E. Bertin, P. Gabriel, M. Raynaud and J.-P. Serre; Revised and annotated edition of the 1970 French original; MR 2867622.

[SGA 4 $4_{\text {II }}$ Théorie des topos et cohomologie étale des schémas. Tome 2, Lecture Notes in Mathematics, 270 (Springer, Berlin, 1972) (in French). Séminaire de Géométrie Algébrique du Bois-Marie 1963-1964 (SGA 4); Dirigé par M. Artin, A. Grothendieck et J. L. Verdier. Avec la collaboration de N. Bourbaki, P. Deligne et B. Saint-Donat; MR 0354653 (50 \#7131).

[Sha64] S. S. Shatz, 'Cohomology of artinian group schemes over local fields', Ann. of Math. (2) 79 (1964), 411-449; MR 0193093 (33 \#1314).

[Sha72] S. S. Shatz, Profinite Groups, Arithmetic, and Geometry, Annals of Mathematics Studies, 67 (Princeton University Press, Princeton, NJ; University of Tokyo Press, Tokyo, 1972); MR 0347778 (50 \#279).

[SP] A. J. de Jong et al., The Stacks Project. http://stacks.math.columbia.edu.

[Toë11] B. Toën, 'Descente fidèlement plate pour les n-champs d'Artin', Compos. Math. 147(5) (2011), 1382-1412 (French, with English and French summaries); doi:10.1112/S0010437X10005245; MR 2834725. 